\title{
Distributed Aspects of the Response to Siphon Touch in Aplysia: Spread of Stimulus Information and Cross-Correlation Analysis
}

\author{
Yang Tsau, Jian-young Wu, ${ }^{a}$ Hans-Peter Höpp, ${ }^{b}$ Lawrence B. Cohen, David Schiminovich, ${ }^{c}$ and Chun Xiao Falk \\ Department of Cellular and Molecular Physiology, Yale University School of Medicine, New Haven, Connecticut 06510 \\ and the Marine Biological Laboratory, Woods Hole, Massachusetts 02543
}

We examined two aspects of the response to siphon stimulation in an attempt to test the hypothesis that the Aplysia CNS functions as a distributed system. First, we estimated the number of central neurons that respond to a light touch to the siphon skin. We made voltage-sensitive dye recordings from the abdominal, pleural, pedal, and cerebral ganglia. From these recordings we estimated that 220 abdominal neurons, 110 pleural neurons, and 650 pedal neurons were affected by the light touch. Thus, the information about this mild and localized stimulus is very widely distributed within the Aplysia CNS. This result allows the possibility that the Aplysia CNS functions as a distributed system. If only a small number of neurons had responded to the touch, it would have supported the conclusion that the gill-withdrawal reflex could be generated by a small, dedicated circuit.

Second, we searched for correlations between the spike times of the individual abdominal ganglion neurons. Two time scales were examined: a millisecond time scale corresponding to the duration of a fast synaptic potential and a seconds time scale corresponding to the duration of the gill-withdrawal movement. Neuron pairs with highly correlated spike activity on a millisecond time scale must be connected by (or have a common input that uses) relatively powerful, fast, excitatory synapses. We expected that this kind of synaptic interaction would be relatively rare in nervous systems that functioned in a distributed manner. Indeed, only $0.3 \%$ of the neuron pairs had correlation coefficients of 0.15 or greater. These correlations accounted for approximately $2 \%$ of the action potentials generated in response to siphon stimulation. Thus, large, fast excitatory synaptic interactions appear to be relatively unimportant. This result is consistent with the hypothesis that the abdominal ganglion functions as a distributed system.

\footnotetext{
Received Aug. 5, 1993; revised Dec. 16, 1993; accepted Dec. 31, 1993.

We thank Barbara Ehrlich, Ray Falk, Chris Hickie, Bruce Lindsey, Bill Ross, Sebastian Seung, and Terry Walters for helpful suggestions. We are grateful to David Senseman for the loan of the 464-element photodiode array and a Silicon Graphics workstation. Vic Pantani and Henrik Abildgaard of the Physiology electronics shop designed and constructed the amplifiers and analog-to-digital converter used to record the output of the diode array. This work was supported in part by Grant NS08437 from NINDS, NIH National Research Service Award NS07102, and an IBM Fellowship.

Present address: Department of 7oology, University of Maryland, College Park, MD 20742.

'Present address: Max-Planck Institut fur Entwicklungsbiologie, D7400 Tubingen, Germany.

'Present address: Department of Physics, Columbia University, New York 10027. Copyright (c) 1994 Society for Neuroscience $0270-6474 / 94 / 144167-18 \$ 05.00 / 0$
}

When the longer time scale was used for the cross-correlograms, a large fraction of the cell pairs had correlated activity because many neurons are activated by the stimulus. It was not possible to interpret the slow correlations in terms of actual synaptic interactions between individual neurons.

Our results are consistent with the possibility that the abdominal ganglion functions in a distributed manner. However, this evaluation is indirect and thus only tentative conclusions can be drawn. Evidence from several sources suggests that the neuronal interactions for generating the Aplysia gill-withdrawal reflex are complex.

IKey words: spread of sensory information, cross-correlation analysis, distributed processing, voltage-sensitive dye, optical recording, Aplysia]

Dedicated neuronal circuits provide a simple conceptual framework for understanding how nervous systems function. However, more recent evidence from both vertebrates and invertebrates has suggested that nervous systems may function in a distributed manner with many neurons involved in even simple behaviors and most neurons functioning in more than one behavior. Optical recordings using voltage-sensitive dyes can simultaneously monitor the action potential activity of many neurons (Salzberg et al., 1977; Grinvald et al., 1981; Zecevic et al., 1989; Nakashima et al., 1992) and may thus be useful in discriminating between these two kinds of neuronal organization. The most complete recordings from the Aplysia abdominal ganglion can detect the spike activity of about $50 \%$ (Wu et al., 1994) of the 900 neurons present in the ganglion (Coggeshall, 1967; Cash and Carew, 1989).

Three kinds of experimental observations may be interpreted to imply that nervous systems function in a distributed manner. The first is that many neurons in different ganglia or brain regions are active during the generation of even relatively simple behaviors (John et al., 1986; Kien and Altman, 1992; Winlow et al., 1992). If many neurons are involved in generating a behavior, most of the individual synaptic interactions would be weak since the presynaptic activity of many cells participate in determining the output of each postsynaptic neuron and no individual neuron should be dominant. Thus, a second kind of observation that is consistent with a distributed organization is the finding that most synaptic interactions in a nervous system are weak. A third kind of observation is that individual neurons participate in several different behaviors (Kristan et al., 1992). We have attempted to evaluate the first two kinds of experimental observations using voltage-sensitive dye recordings from the Aplysia californica CNS during the response to a light touch to the siphon skin. 
Earlier optical recordings suggested that 200-300 abdominal ganglion neurons were activated by a light touch (Zecevic et al., 1989; Nakashima et al., 1992). However, in those experiments only the abdominal ganglion was retained. Here, we report similar measurements of spike activity in the abdominal, pleural, cerebral, and pedal ganglia in a preparation where the pleuralabdominal connectives remained intact.

Cross-correlation analysis can be used to detect and partially characterize neuron interactions. Fast correlations (a time scale of milliseconds) have been used to evaluate the type and strength of the synaptic interactions between pairs of neurons (Perkel et al., 1967; Moore et al., 1970; Toyama et al., 1981; Lindsey, 1982; Aersten and Gerstein, 1985; Tam et al., 1988; Eggermont, 1990). The ability to detect these correlations is dependent on the numbers of spikes in the recordings. In contrast to previous correlation analyses of records including thousands of spikes from each neuron, the duration of the optical recordings is limited and the mean number of spikes is low. Therefore, only relatively large correlations could be detected. On the other hand, optical recordings detect the activity of many neurons and thus a large number of pairs can be examined to obtain a reasonably complete evaluation of the number of neurons pairs connected by large, fast, excitatory synapses.

Because preliminary analysis showed that only a small fraction of neuron pairs had apparently significant correlations, it was possible that those correlations might have occurred by chance. To evaluate this possibility we generated randomized data sets from the experimental data and carried out the analysis on these randomized sets. The optical measurements from the abdominal ganglion record the activity of more than 100 neurons, and thus the pairwise cross-correlation analysis for one data set involves the evaluation of correlograms from about 10,000 pairs of neurons. With the 10 randomized data sets there were 100,000 correlograms. We used two methods for evaluating these correlograms. The first was visual inspection of the shape of selected correlograms and the second was the use of the computed correlation coefficient.

Multicellular recordings from the Aplysia abdominal ganglion show that the activity of a large number of neurons is highly correlated with the sensory stimulus. Most neurons have greatly increased activity in the seconds following the stimulus (Zecevic et al., 1989; Nakashima et al., 1992). Therefore, on a time scale of several seconds we expected to find large correlations in the activity of many neuron pairs. Thus, cross-correlograms were also constructed over a slow time scale (a $6 \mathrm{sec}$ interval).

Preliminary reports of these experiments have appeared previously (Schiminovich et al., 1989; Cohen et al., 1990).

\section{Materials and Methods}

The isolated siphon preparation (Kupfermann et al., 1971) was used for the experiments that provided the data for cross-correlation analysis. This preparation (Fig. 1) includes only the abdominal ganglion, the siphon, gill, and connecting nerves. For the experiments measuring the response to touch in the pleural, pedal, and cerebral ganglia, we used a preparation that included these ganglia while maintaining their connection to the abdominal ganglion via the pleural-abdominal connectives. Five recordings were made in these experiments. The first and last were from the abdominal ganglion, the second was from one of the pedal ganglia, the third from one of the pleural ganglia, and the fourth from one of the cerebral ganglia. The only large central ganglion that we did not examine was the buccal ganglion. In both preparations the siphon and gill were peripherally isolated and thus, central neurons were necessary participants in generating the behavioral output. Mechanical stimulation was delivered to the siphon skin via a computer controlled motor that moved a $0.6 \mathrm{~mm}$ glass rod with a rounded tip. The drawing at the lower right of Figure 1 shows schematically the relative positions of the image of the abdominal ganglion and the 464-element photodiode array. The drawing of the cell bodics is only schematic and the position of the array relative to the ganglion is only approximate. The animals weighed between 5 and $20 \mathrm{gm}$. The preparations were bathed either in seawater or artificial seawater containing $460 \mathrm{~mm} \mathrm{NaCl}, 10 \mathrm{~mm} \mathrm{KCl}$, $11 \mathrm{~mm} \mathrm{CaCl}_{2}, 55 \mathrm{~mm} \mathrm{MgCl}_{2}$, and $10 \mathrm{~mm}$ Tris- $\mathrm{HCl}, \mathrm{pH}$ 7.6. The ganglia were not desheathed. The chamber temperature was kept at $15 \pm 1{ }^{\circ} \mathrm{C}$.

\section{Optical recording}

We used two voltage-sensitive pyrazolone-oxonol dyes: NK304I (Grinvald et al., 1982; obtained from Nippon Kankoh Shikiso Kenkyusho Co. Ltd., Okayama, Japan) and its diethyl analog, JPW 1131 (obtained from Dr. Leslie Loew, Department of Physiology, University of Connecticut, Farmington, CT). These dyes were first suggested and synthesized by Rina Hildesheim and Amiram Grinvald as RH155 and RH479. The ganglia were stained with a dye concentration of $0.15 \mathrm{mg} / \mathrm{ml}$ following the staining protocol of Nakashima et al. (1992).

The optical recordings in the experiments with suction electrode stimulation of the siphon skin (A188 and A191) were made using 124 elements of a $12 \times 12$ array (Centronic Inc., Newbury Park, CA). All other experiments were carried out with a 464 -element photodiode array (in a $24 \times 24$ grid with the corner elements omitted). With the 124pixel photodiode array the most complete recordings monitored the activity of about $30 \%$ of the neurons (Zecevic et al., 1989); the best recordings made with a 464-pixel array (used in later experiments) were estimated to be about 50\% complete (Wu et al., 1994). These estimates came from experiments where we attempted to activate all of the neurons in the abdominal ganglion by electrical stimulation of the five largest nerves and connectives. Thus, the best experiments would record the activity of about 450 of the 900 neurons (Coggeshall, 1967; Cash and Carew, 1989) in the abdominal ganglion of young adults. While activity in this many neurons can be detected in response to maximal stimulation, substantially fewer neurons are activated by a light touch to the siphon skin. We used a sampling rate of one data point per msec for the $12 \times 12$ array and one point per $1.6 \mathrm{msec}$ for the $464 \mathrm{clcment}$ array. For additional details, see Zecevic et al. (1989), Falk et al. (1993), and $\mathrm{Wu}$ and Cohen (1993). The raw data from the optical recordings consist of cell-body spike signals on photodiode outputs. A sorting procedure is required to determine the activity of individual neurons from this data. For examples and descriptions of this process, see London et al. (1987), Zecevic et al. (1989), and Falk et al. (1993).

Pharmacologic effects of the voltage-sensitive dye or phototoxic effects of illumination could alter the threshold and affect the number of neurons that respond to the stimulus (Wu and Cohen, 1993). We think that such effects are small; if they exist they are likely to be in the direction of decreasing the response. In some experiments the gill withdrawal in response to the touch was not affected by the dye staining procedure or by the illumination (data not shown). At higher dye concentrations where such effects have been observed (Zecevic et al., 1989; Nakashima et al., 1992) they were in the direction of a decreased response.

We have not attempted to relate the optically detected neurons to previously identified neurons. Some difficulties in determining these relationships were discussed in Wu et al. (1994).

\section{Cross-correlation analysis}

Cross-correlograms. Cross-correlograms between pairs were calculated by accumulating a histogram of time differences between the occurrence of spikes in the two cells of a pair. The time of the spikes of one of the cells is set at zero time, the time difference between those spikes and the spikes in the second cell is displayed on the $\mathrm{x}$-axis, and the number of occurrences on the y-axis. For the fast time scale results illustrated in Figures 6-9, we used four data point time bins for a \pm 64 data point time interval surrounding the spike times of one of the pair of cells. Other time intervals were also tested $( \pm 16, \pm 32$, and \pm 128 data points). We computed the corrclation cocfficicnt, $r_{x k}(t)$, for cach pair using Equations 1 and 2 (Christakos, 1984).

$$
\begin{aligned}
r_{s y}(\tau) & =\frac{R_{x y}(\tau)}{\sqrt{R_{x x}(0) R_{y y}(0)}} \\
R_{x y}(\tau) & =\sum_{n=0}^{T} X(n) Y(n+\tau)
\end{aligned}
$$




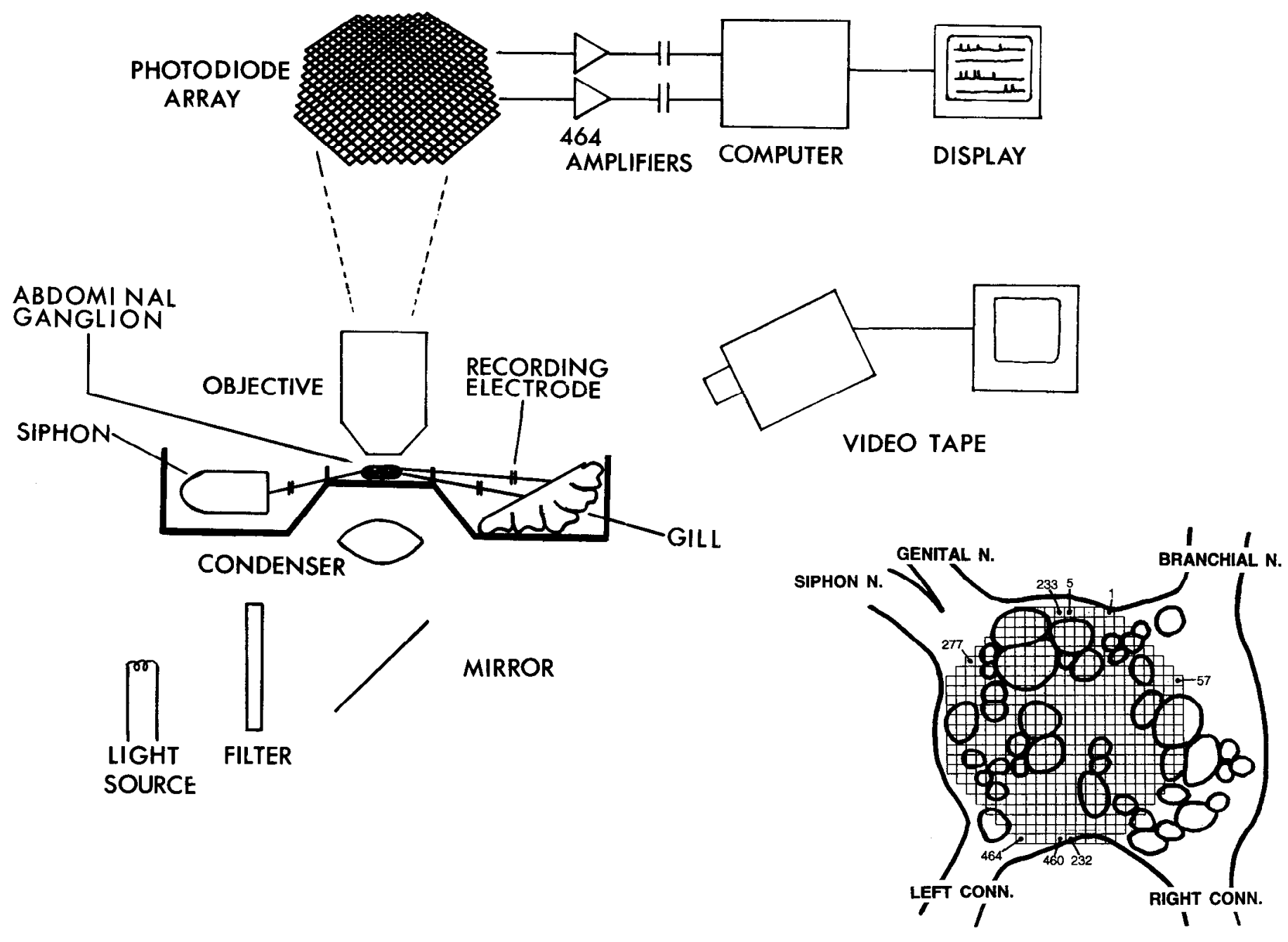

Figure 1. Schematic drawing of the preparation and apparatus. Light from a tungsten halogen lamp was passed through a $720 \pm 25 \mathrm{~nm}$ interference filter and focused on the preparation using a modification of Kohler illumination. The condenser iris was opened so that the condenser numerical aperture would equal the objective numerical aperture. A $12 \times 12$ or a 464-element pholodiode array was placed at the plane where the objective forms the real, inverted image. The photodiode array records the light transmitted by the ganglion. The outputs from each diode were individually amplified. The signals were digitized by a dual analog-to-digital converter and stored in a Motorola 68000 VME bus computer. The drawing at the lower right shows schematically the relative positions of the image of the abdominal ganglion and the 464-element photodiode array. The numbering scheme used for the 464-element array is indicated on the drawing.

The numerator of Equation 1 is the number of coincident spikes using a time delay $t$, and the denominator is the square root of the product of the total number of spikes in the two neurons. The number of coincident spikes was the largest number found in a running 12 data point window that was passed through the total interval. Window widths of 4 and 20 data points were also used. $R_{r r}(t)$ is the cross-correlation function of two spike $d$ functions $X(n)$ and $Y(n+t), R_{x x}(0)$ and $R_{y, y}(0)$ are the spike counts of the two neurons, and $T$ is the duration of the recording. Figure 2 illustrates the raster diagrams, cross-correlograms, and correlation coefficients for four example pairs of neurons. The timing of the spikes in the first three pairs was fabricated. The fourth pair is the data from two neurons in experiment A206 whose cross-correlogram is also shown as the third correlogram in Figurc 6.

In addition to the correlation coefficient we also evaluated the crosscorrelograms by visual inspection for both excitatory and inhibitory effects. Where apparent correlations were detected, we reexamined the original data to check whether the apparent correlations might result from an analysis artifact. In each instance, apparent inhibitory interactions were actually the result of an artifact that occurred when erasing the spike signal from one neuron inadvertently erased data from a second neuron. No real inhibitory interactions were detected using a fast time base.

Generation of random data sets. Because we examined a large number of cell pairs, we needed to distinguish between cross-correlations that resulted from real neuronal interactions and those that resulted by chance. We compared the correlation coefficients from the original experimental data sets with scores obtained using random data sets generated from the original data. The result of this kind of comparison depends on the method used for generating the random sets. If one simply maintains the total number of spikes constant between the experimental and random sets but allows both the assignment to neurons and the assignment of spike times to be random, then a random set is generated with a greatly reduced possibility of chance correlations. This occurs because both the spikes per neuron and the spike times are highly grouped in the experimental data and this grouping increases the possibility for the chance occurrence of correlated spike times. To make a fair comparison, the random data set should have the same grouping of spikes in cells and spikes in time as the experimental data set.

We generated random sets by replacing each experimental spike time in the data set with a new time chosen randomly within a window around the real time. We compared the results of this process using windows of 1024,128 , and 64 data points. Using larger window widths in the randomization increased the distortion of the temporal grouping in comparison with the experimental data. The difference in the number of pairs with large scores in the experimental and random data sets decreased as the window width decreased. However, the difference be- 


\section{Raster \\ Diagrams}

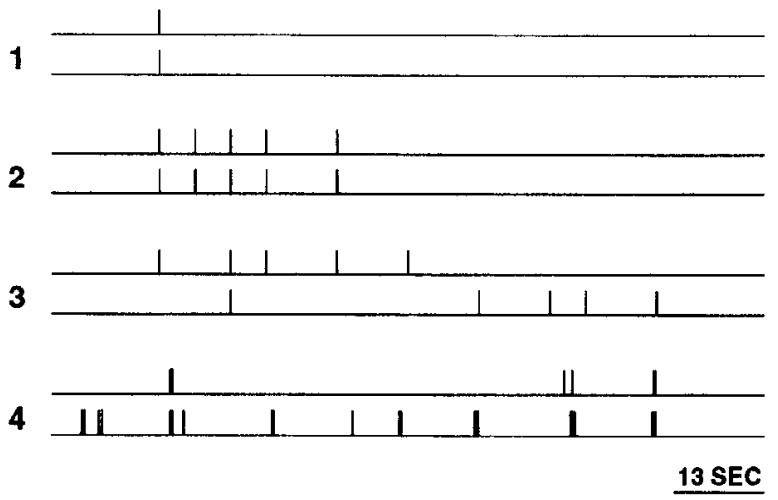

CrossCorrelograms
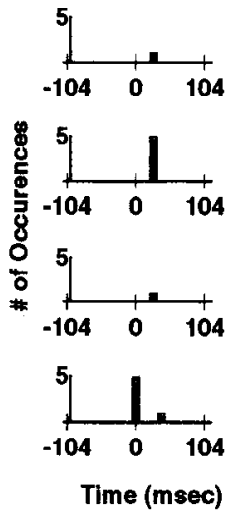

Correlation

Coefficients

1

1

0.2

0.39

Figure 2. Illustration of firing patterns (raster diagrams), cross-correlograms, and correlation coefficients for four example cell pairs. The crosscorrelograms between pairs were calculated by accumulating a histogram of time differences between the occurrence of spikes in the two cells of a pair. For calculating the correlation coefficient the number of coincident spikes was the number present in a running 12 data point window. The top two cell pairs have correlation coefficients of 1.0 , the third pair a correlation coefficient of 0.2 , and the bottom pair 0.39 . The spike times for the first three pairs were fabricated; the times for the fourth pair come from two neurons in experiment A206. Because of the condensed time scale of the raster diagram, it is not possible to discern the delay between the spikes in the first thrce pairs. In the fourth pair, the thicker lines represent multiple spikes with relatively short interspike intervals.

tween 128 and 64 data points was small, suggesting that a window width of 64 data points did not substantially distort the temporal grouping of activity. Tests with fabricated data (e.g., the first three pairs of Fig. 2) showed that randomization with a 64 data point window did greatly reduce the cross-correlation scores of highly correlated pairs.

The calculations were done on either a Motorola VME-bus computer or on a Silicon Graphics Indigo workstation using MATHEMATICA (Wolfram Research, Champaign, IL; version 2.1) on the workstation. The data displays were generated using MATHEMATICA. The sourcc codc is available from the authors.

\section{Results}

Spread of sensory information in the central ganglia of Aplysia

We made optical recordings from the abdominal, pleural, pedal, and cerebral ganglia during application of a light touch $(2 \mathrm{gm}$, $600 \mathrm{msec}$ ) to the siphon skin. These stimuli elicited a gill-withdrawal reflex in each trial. The results from one experiment are illustrated in Figure 3. Four panels are shown, the first for the abdominal ganglion, the second for one pleural ganglion, and the last two for the results from one pedal ganglion. At the top are raster diagrams of the spike activity. Each horizontal line represents one neuron; each vertical tick mark, one action potential. We detected activity in 81 neurons in the abdominal ganglion, 46 neurons in the pleural ganglion, and 181 neurons in the pedal ganglion. We have divided the neurons of the pedal ganglion into three groups. In the first group (third panel) are the neurons whose activity increased by more than $30 \%$ follow- ing the siphon stimulus. In the second and third groups (fourth panel) are the neurons whose activity decreased by more than $30 \%$ and the neurons whuse activity changed by less than $30 \%$. Below the raster diagrams in Figure 3 are histograms of the total number of spikes in $820 \mathrm{msec}$ time bins from each panel. At the bottom are shown the gill contractions for the three trials.

From both the raster diagrams and the histograms it is clear that the overall response differs from ganglion to ganglion. The response in the pleural ganglion is briefer and the response in the pedal ganglion more prolonged than the response in the abdominal ganglion. In addition, in all three experiments there was more background activity (before the stimulus) in the pedal ganglion than in the abdominal or pleural ganglia. While almost all of the abdominal and pleural neurons were activated by the stimulus, this was not true for the neurons in the pedal ganglion. Thirty-one of the 181 pedal neurons are separated into a group whose activity appears to be unaffected by the stimulus. Nonetheless, most of the pedal neurons were either activated or inhibited by the very light and localized touch to the siphon skin.

The numbers of affected neurons from the experiment of Figure 3 are shown in the first column of Table 1 . To make comparisons across experiments we normalized the number of affected neurons to the number in the abdominal ganglion. The second column shows the mean results from three experiments. In two recordings from the cerebral ganglion the number of active neurons was small and little or none of the activity was stimulus related; this ganglion is not included in Table 1. For

\footnotetext{
Figure 3. Spike activity in the abdominal, pleural, and pedal ganglia during a light mechanical touch to the siphon skin. At the top are the raster diagrams of spike activity; each horizontal line represents one neuron, and each vertical tick mark one action potential. Spike activity was detected in a relatively large number of pedal neurons; for clarity of presentation we divided the pedal neurons into threc groups. The third column contains neurons whose poststimulus spike frequency was more than 1.3 times the prestimulus frequency. The fourth column contains two groups of pedal neurons: those whose poststimulus activity was less than 0.7 of the prestimulus activity (top), and those neurons whose activity changed by less than $30 \%($ bottom). Below the raster diagrams are histograms of the total number of spikes in 820 msec time bins. At the bottom are shown recordings of the gill contractions obtained by measuring the gill area from videotape recordings. The recordings from the three ganglia were made at 15 min intervals; the trials were carried out in the order abdominal, pedal, pleural. In this and the subsequent two figures the dashed lines are placed at the beginning of the current steps that drove the mechanical toucher (Figs. 3, 4) or the beginning of the first current step passed across the cell membrane via an intracellular microelectrode (Fig. 5).
} 
Abdominal G.

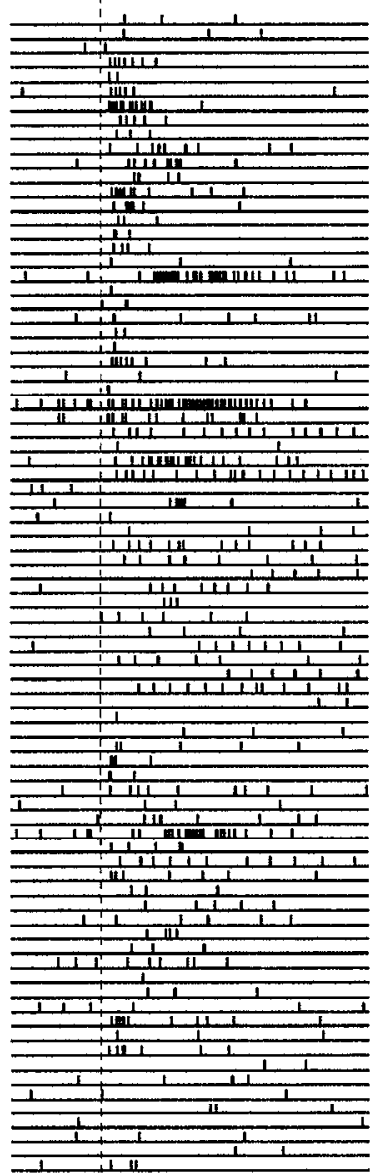

Pleural G.

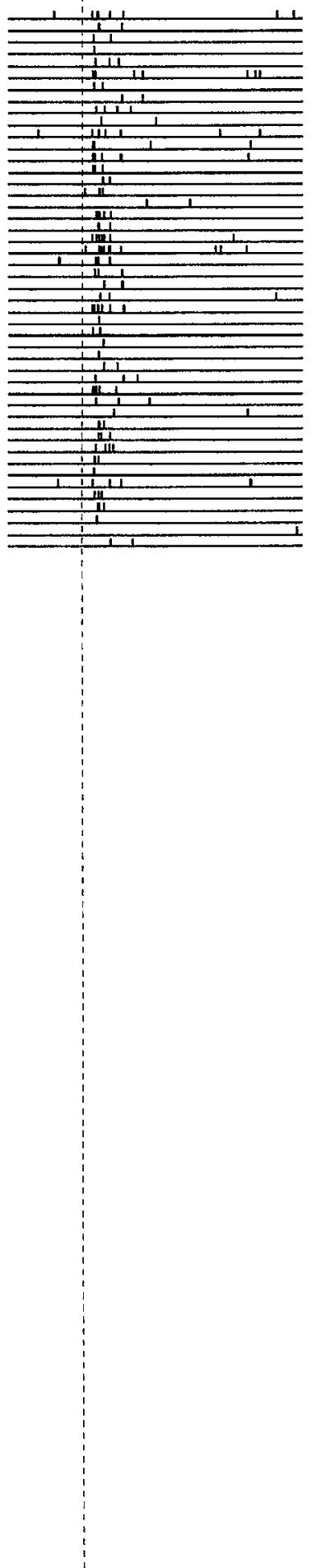

Pedal G.

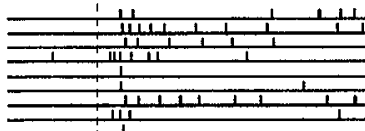

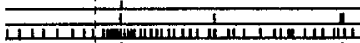
IIIIII 111111 $\frac{1}{1} \frac{1}{1} \frac{1}{1}$

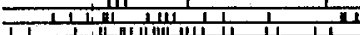
II 工 I

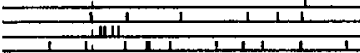
$\frac{1}{11} \frac{1}{111} \frac{1}{11}$

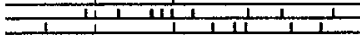

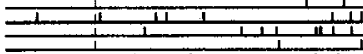
I I I IIIII I IIII IIIIIII IIIII

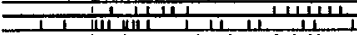
$=\frac{1}{1}{ }_{11}$

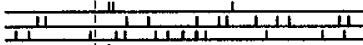
$\frac{11}{1}$

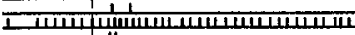

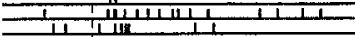

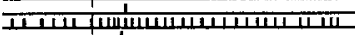
$\frac{11}{1 ! 1}$

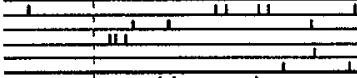

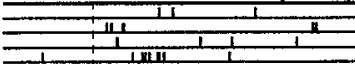
三

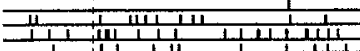

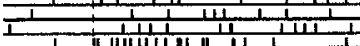
$\frac{1}{1}=\frac{1}{1}$ 패

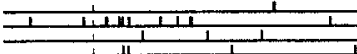

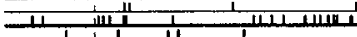

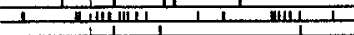

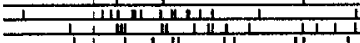

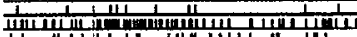
I.

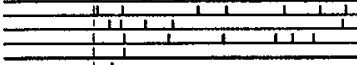

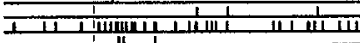

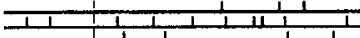

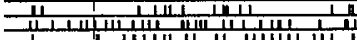
I I I INUWI IIIIIII $\frac{11}{1+111 ! 1}$
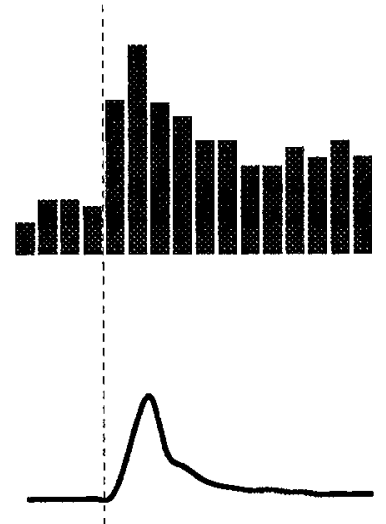

(continued)

\section{Inhibited}

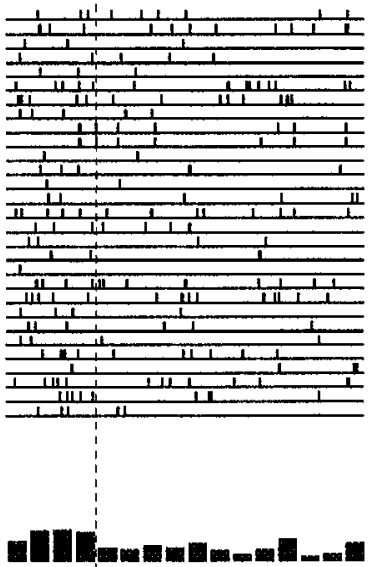

\section{No Change}

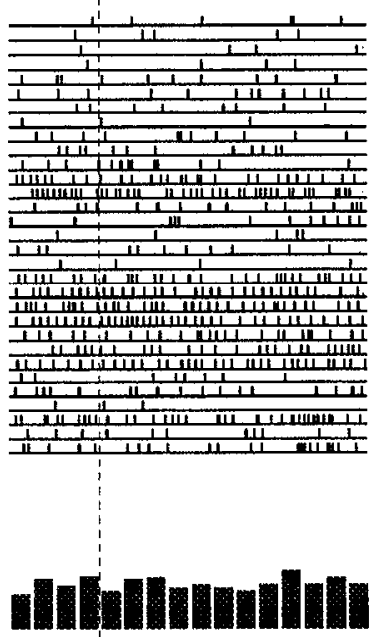

13 SEC 


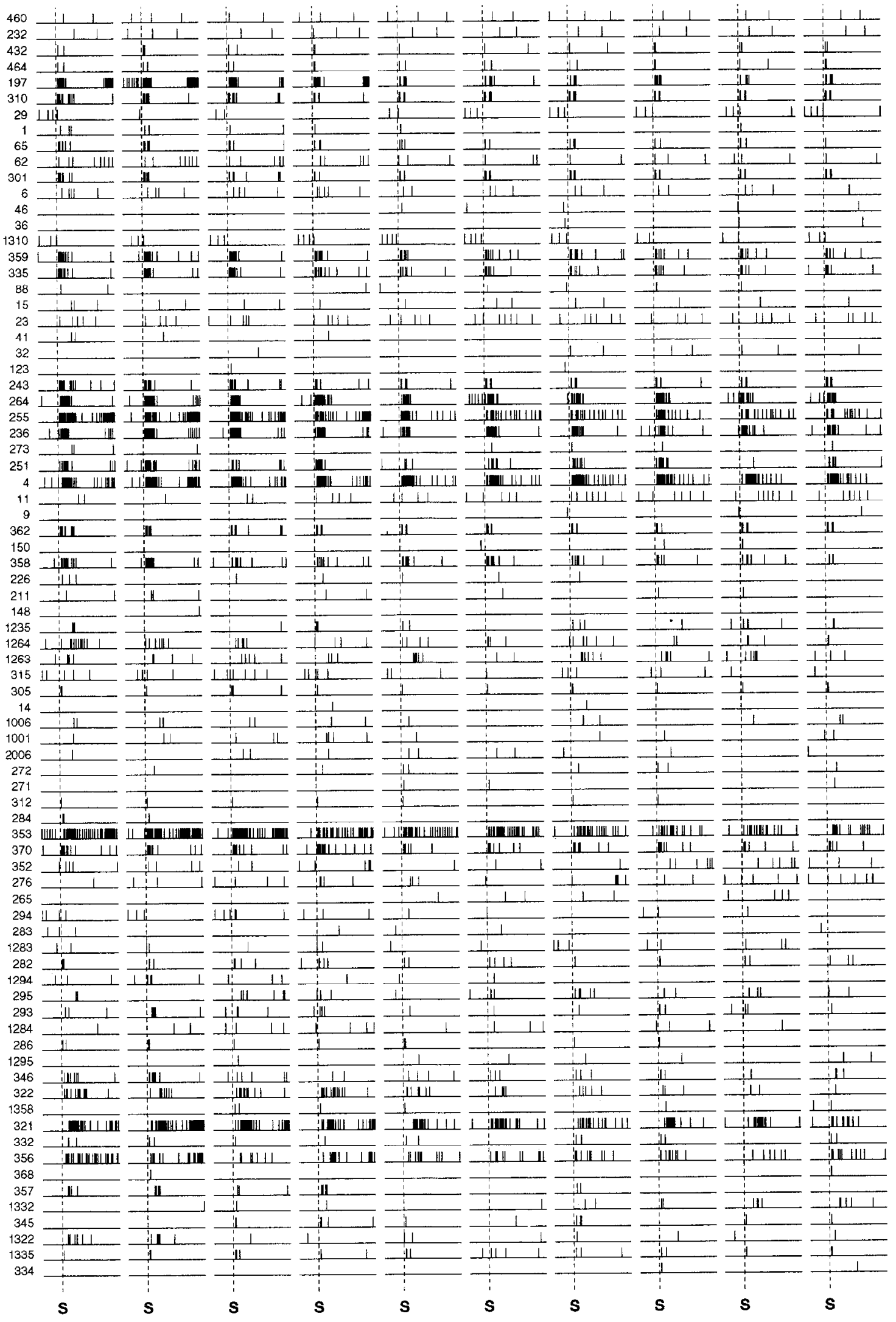

Figure 4. Raster diagrams of action potential activity detected in an abdominal ganglion during 10 presentations of a light touch separated by 10-15 min intervals. This is one of the four data sets used for the fast cross-correlation analysis. Each vertical panel shows a 13 sec recording. In this figure and in Figure 5 the numbers to the left of each line in the raster diagram are identification numbers derived from their signal locations 


\begin{tabular}{|c|c|c|c|c|c|}
\hline Ganglion & $\begin{array}{l}\text { \# of } \\
\text { neurons } \\
\text { (Fig. 3) }\end{array}$ & $\begin{array}{l}\text { Normal- } \\
\text { ized } \\
\text { mean \# } \\
(n=3)\end{array}$ & $\begin{array}{l}\text { Correction } \\
\text { for } \\
\text { symmetry }\end{array}$ & $\begin{array}{l}\text { Estimated } \\
\text { actual \# }\end{array}$ & $\begin{array}{l}\text { Percent- } \\
\text { age }\end{array}$ \\
\hline Abdominal & 81 & 1.0 & 1.0 & 220 & 25 \\
\hline Pleural & 46 & 0.25 & 0.5 & 110 & 15 \\
\hline Pedal & 150 & 1.47 & 2.94 & 650 & 20 \\
\hline
\end{tabular}

the pedal ganglion we only included neurons which were affected by the stimulus.

We made measurements from only one of the pair of pedal and pleural ganglia. If the spread of activity from the abdominal ganglion were symmetrical, then the number of active neurons in the paired ganglia would be twice that which we measured in an individual ganglion. This correction is shown in the third column of Table 1. This column is our best estimate of the proportions of active neurons in the different ganglia. The last columns are the estimated actual number and percentage of activated neurons in the three ganglia after additional corrections described in the Discussion.

\section{Cross-correlation analysis}

Cross-correlation analysis was carried out on data sets from five isolated siphon preparations. In each experiment 6-10 trials were recorded with a total recording time of between 80 and $130 \mathrm{sec}$. In each set the data from all of the recorded trials was used for the cross-correlation analysis. In all five data sets almost all neurons show an obvious overall correlation of spike activity with the stimulus. Two of the data sets are shown in Figures 4 and 5; these are from experiments A207 and A217. Each vertical panel represents the results of one trial. The dashed lines with the label " $\mathrm{s}$ " in the two figures indicate the time of the beginning of the stimulus (light touch to the siphon or microelectrode current). A portion of an additional data set (A206) is shown in Figure 2 of $\mathrm{Wu}$ et al. (1994). In A206 and A207 the gillwithdrawal reflex was elicited by a mechanical touch to the siphon skin. Two additional data sets, A188 and A191, come from experiments where suction electrode stimulation of the siphon skin was used to elicit the gill withdrawal.

Table 2 indicates the number of neurons, cell pairs, the mean number of spikes per cell and the stimulus type for the five data sets.

In experiment A217 (Fig. 5) a single neuron, 283 (top trace; probably L10), in the abdominal ganglion was stimulated six times with a microelectrode, giving rise to 19 action potentials on each occasion. The locations of the group of neurons that were active at the time the driven cell was stimulated (early response) are shown in the top right panel of Figure 5. These neurons were found in a restricted region in the top central portion of the ganglion. The locations of the group of neurons that were active during the delayed burst (late response) are shown in the bottom right panel. While it is clear that some neurons are part of both groups, the second group includes more

\begin{tabular}{|c|c|c|c|c|}
\hline $\begin{array}{l}\text { Prepa- } \\
\text { ration }\end{array}$ & $\begin{array}{l}\text { \# of } \\
\text { neurons }\end{array}$ & \# pairs & $\begin{array}{l}\text { Mean \# } \\
\text { of spikes }\end{array}$ & Stimulus \\
\hline A 188 & 92 & 4186 & 43 & Suction electrode \\
\hline A191 & 125 & 7750 & 67 & Suction electrode \\
\hline A206 & 149 & 11,026 & 32 & Touch \\
\hline A207 & 79 & 3081 & 46 & Touch \\
\hline $\mathrm{A} 217$ & 63 & 1953 & 34 & Microelectrode \\
\hline
\end{tabular}

neurons and these neurons are found over a larger area of the ganglion. This area is, however, restricted in comparison with the neurons that respond to siphon touch, which are found in all areas of the ganglion (Fig. 8 of Falk et al., 1993).

\section{Fast correlations}

Cross-correlation analysis with a fast time scale was carried out on the first four data sets from Table 2. Figure 6 illustrates 24 of the 11,026 cross-correlograms from experiment A206. In the left panel are the 12 correlograms with the largest correlation coefficients. On the right are 12 correlograms with scores between the 101st and 112th largest. Several of the cross-correlograms in the left panel involve a substantial number of identically timed intervals; for example, the ninth correlogram has a peak of 17 identically timed occurrences. These numbers suggest that the correlations might be significant. This is less often true of the correlograms in the right panel. None of the large number of cross-correlograms with correlation coefficients of less than 0.1 appeared to be significant. Two kinds of correlations are seen in the left panel. The first, third, fourth, sixth, and ninth correlograms have a peak in the interval surrounding $0 \mathrm{msec}$, suggesting the existence of a common input for the pair of neurons (Moore et al., 1970). The second, fifth, seventh, and eighth correlograms have a peak displaced to one side of $0 \mathrm{msec}$, with offsets in the range of one or two synaptic delays, suggesting that one of the pair may be driving the other. The vast majority of the 11,026 pairs have small correlation coefficients.

The criteria we used to determine whether the correlations were significant or simply due to chance occurrences was to compare these cross-correlograms with those obtained from randomized data sets derived from the original data. The left panel of Figure 7 illustrates the cross-correlograms with the largest correlation coefficients from one such random data set. Both the correlation coefficients and the number of identically timed occurrences are smaller in the randomized data set than in the experimental data (Fig. 6). To estimate the range of correlation coefficients expected from random sets we made 10 randomized data sets from each experimental data set. If the number of pairs with large scores from the experimental data set falls within the range determined by the 10 randomized sets then we would conclude that there were no significant correlations in the experimental data. On the other hand, if the number of pairs from the experimental data set were larger than the largest of the 10 random sets, we would conclude that there was a significant excess. 


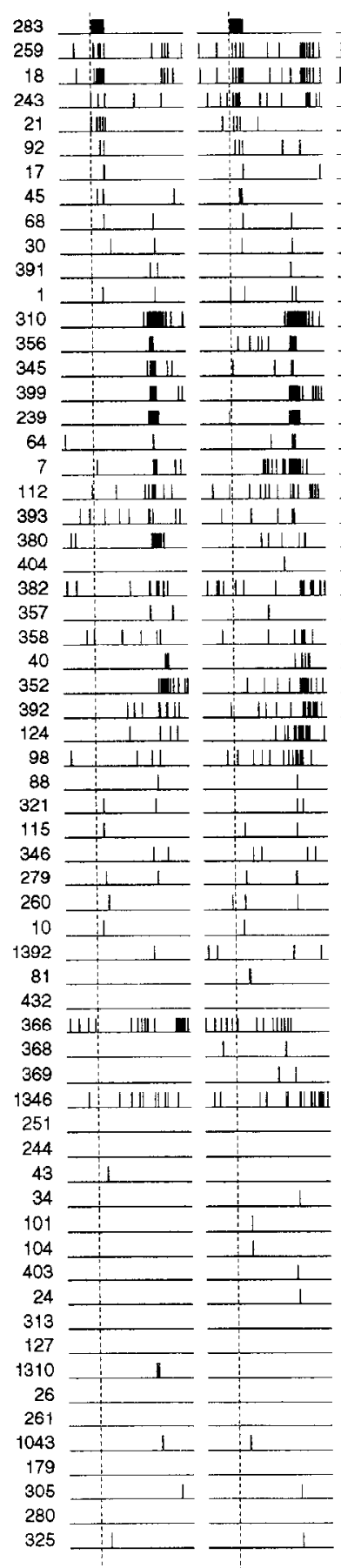

s s

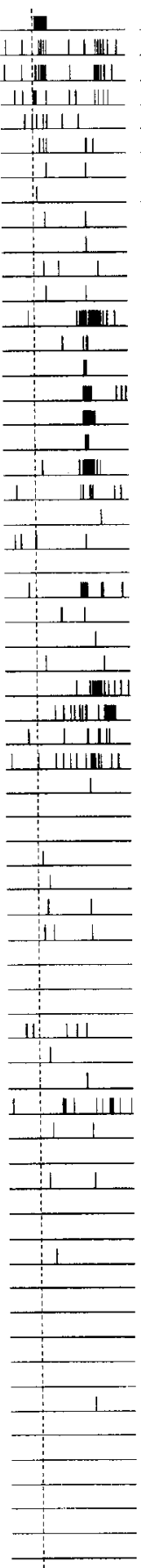

s
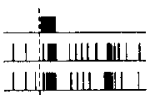

III) 1+1| III!|

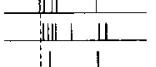

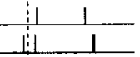

$\frac{1}{1}$
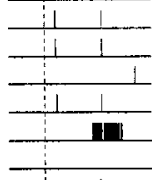

$\frac{1}{1}: \frac{1}{1}$

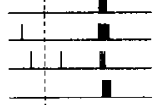

$$
\text { . }
$$

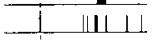

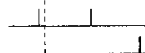
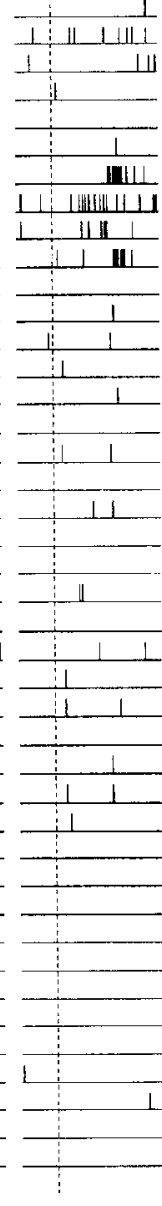

s

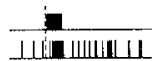

Lin Hul|

$\frac{\text { ill }}{\text { iلl }}$

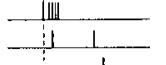

\begin{tabular}{c:c}
1 & 1 \\
\hline 11 \\
\hline 1
\end{tabular}

\begin{tabular}{c}
11 \\
\hline 111 \\
\hline 1
\end{tabular}

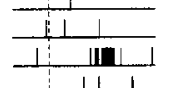

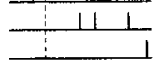

\begin{tabular}{c|c}
\hline$: 1$ \\
\hline$\vdots 1 \mathrm{I} \perp 1$ \\
\hline 141
\end{tabular}

\begin{tabular}{r:l}
\hline 1 & 1 \\
\hline 1 & 1
\end{tabular}

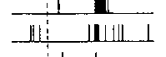

\begin{tabular}{l:l}
11 \\
\hline 111
\end{tabular}
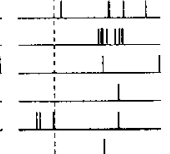

\begin{tabular}{c:c}
\hline+1 \\
\hline $111 \perp$
\end{tabular}
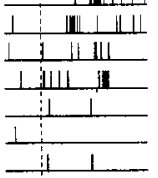

\begin{tabular}{c:c}
11 \\
\hline 111 \\
\hline 111
\end{tabular}

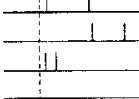

\begin{tabular}{c:}
1 \\
\hline \\
\hline
\end{tabular}
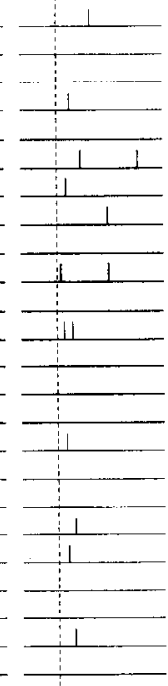

s

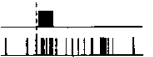

HI Lill IIII!

上揭比

\begin{tabular}{ccc}
\hline & 1 \\
\hline$\vdots$ & 1 & \\
\hline & 1 & \\
\hline
\end{tabular}
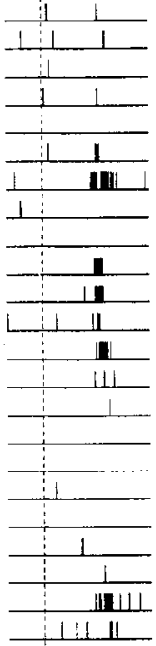

$11: 1$

$$
\begin{array}{c:c}
\hline & 1 \\
\hline 1 & 1 \\
\hline 1 & 1
\end{array}
$$

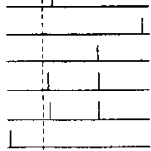

\section{LATE RESPONSE}

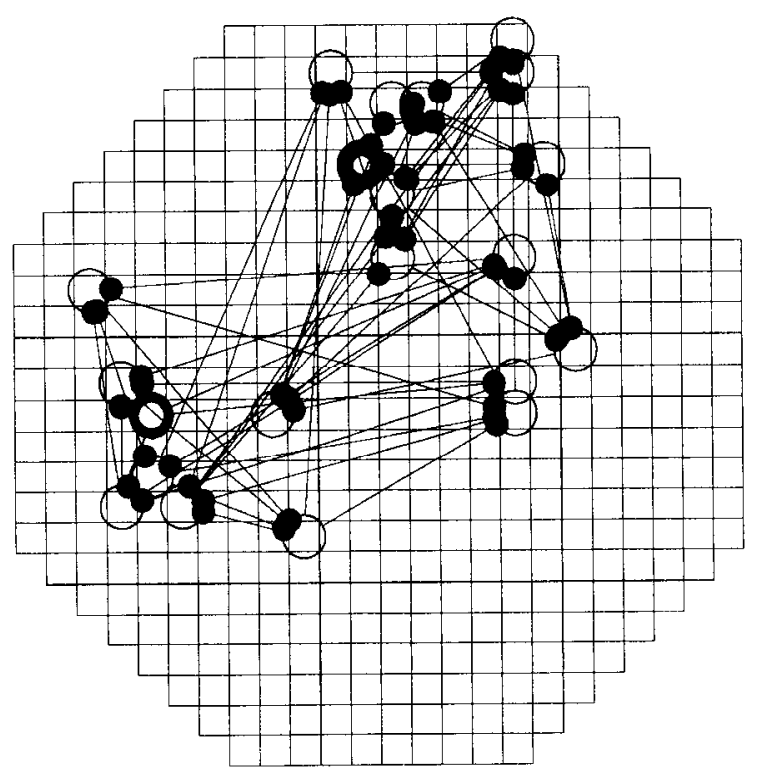

s
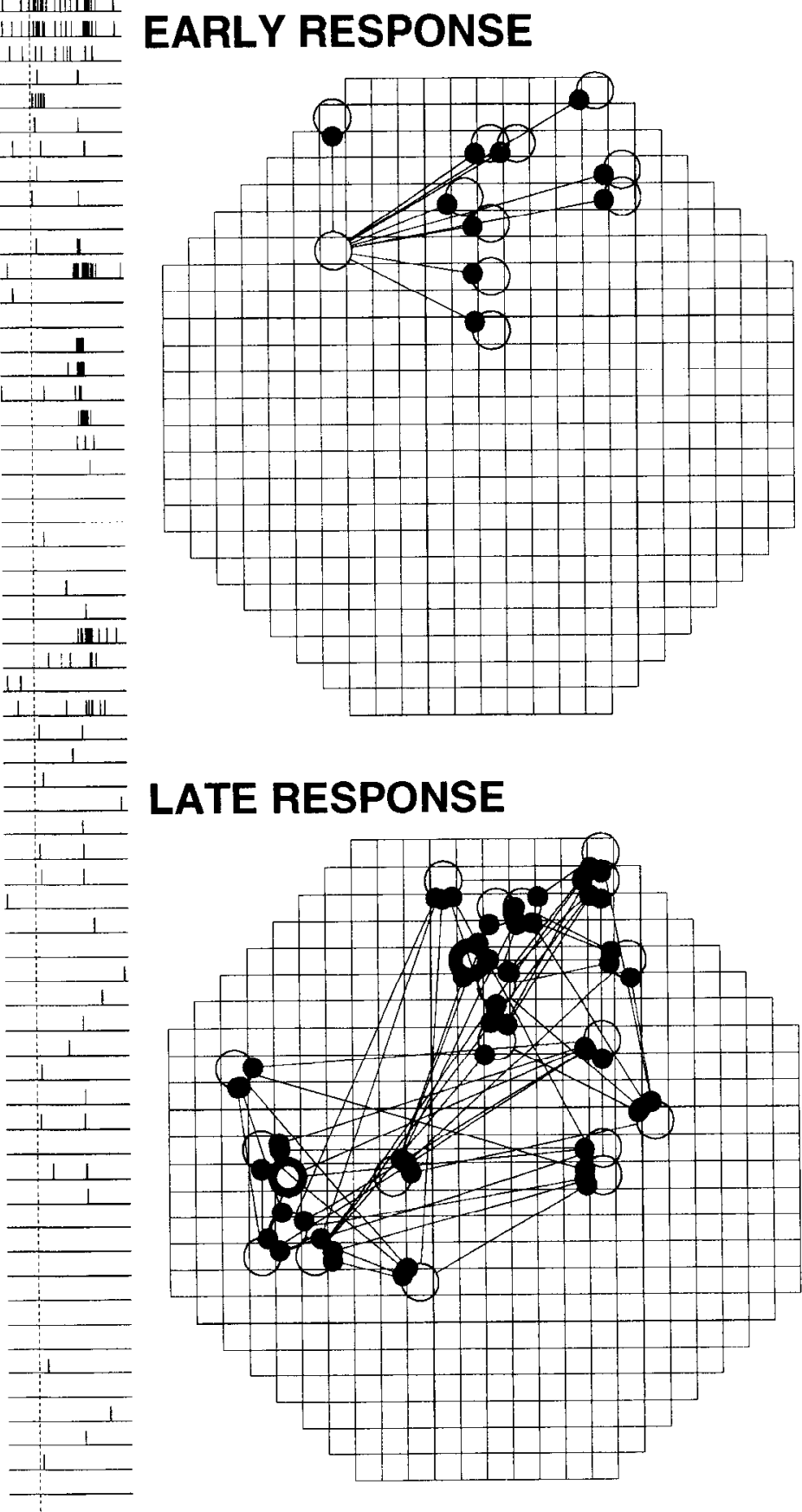

\section{SEC}

Figure 5. The right panel illustrates raster diagrams of action potential activity detected in an abdominal ganglion as a result of six intracellular microelectrode stimulations of neuron $\# 283$ (possibly L10; top trace). In each triai this neuron was driven to make 19 action potentials. No gill movements resulted from this stimulation. The locations of the activated neurons are shown in the right panels. At the top are the locations of the driven neuron and the additional neurons that were active during the period during which the driven neuron was stimulated (early response). At the bottom are the locations of neurons that participated in a delayed response. The neurons that are part of the early response are located in a more restricted area of the ganglion than the neurons that participated in the delayed response. Arbitrarily chosen examples of synaptic interactions that could explain the slow correlations seen among the neurons are shown. For the early response we show the connections obtained under the assumption that the driven cell makes a direct synaptic contact with each of the activated neurons. For the delayed response we used the temporal ordering of spike activity from the cross-correlograms to generate the synaptic connections. The cell that fired earliest is indicated by the thick circle; the cell that fired last is indicated by the thickest circle. Each neuron was synaptically connected to the three neurons that followed it most closely in time. Thus, each neuron had synaptic interactions with about $8 \%$ of the neurons in the group. Unfortunately, these examples were rather arbitrarily chosen from many possible maps of synaptic connections. Because slow correlations are found between many pairs of neurons, and because the correlations are slow, it is not possible to know which correlations represent actual synaptic connections. Experiment number A217. 
1-12; EXPERIMENTAL corr. coeff.
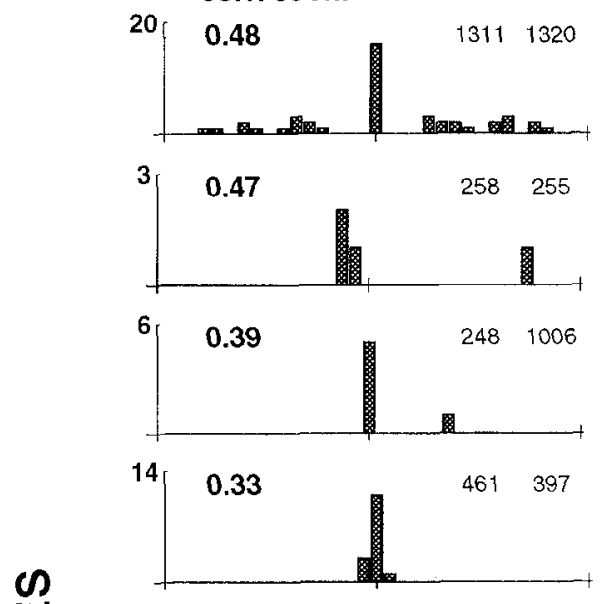

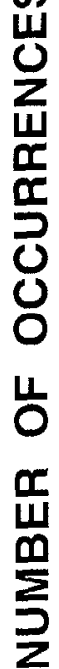
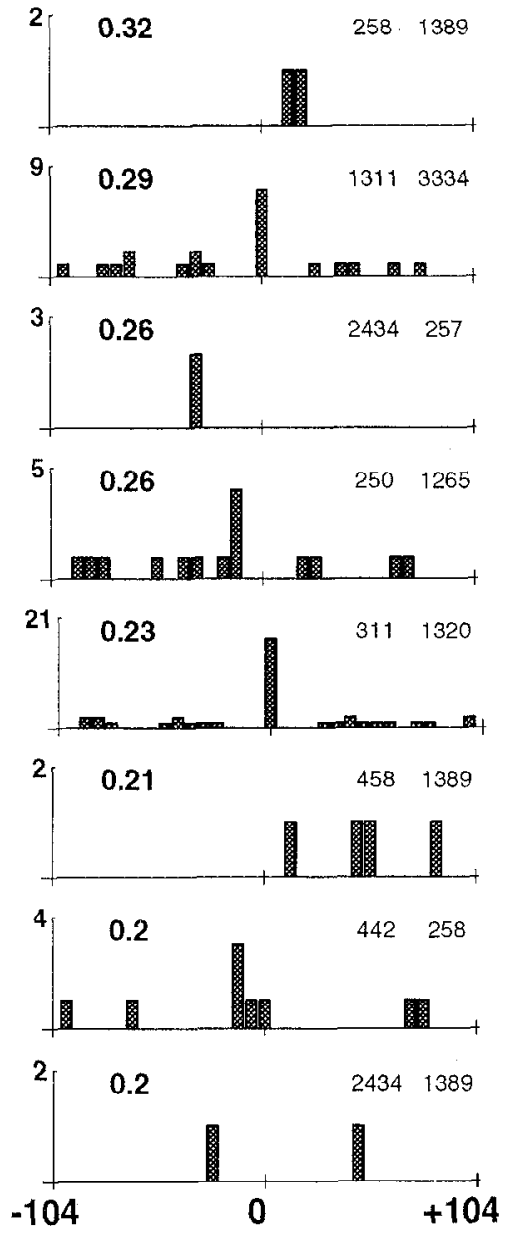

101-112; EXPERIMENTAL
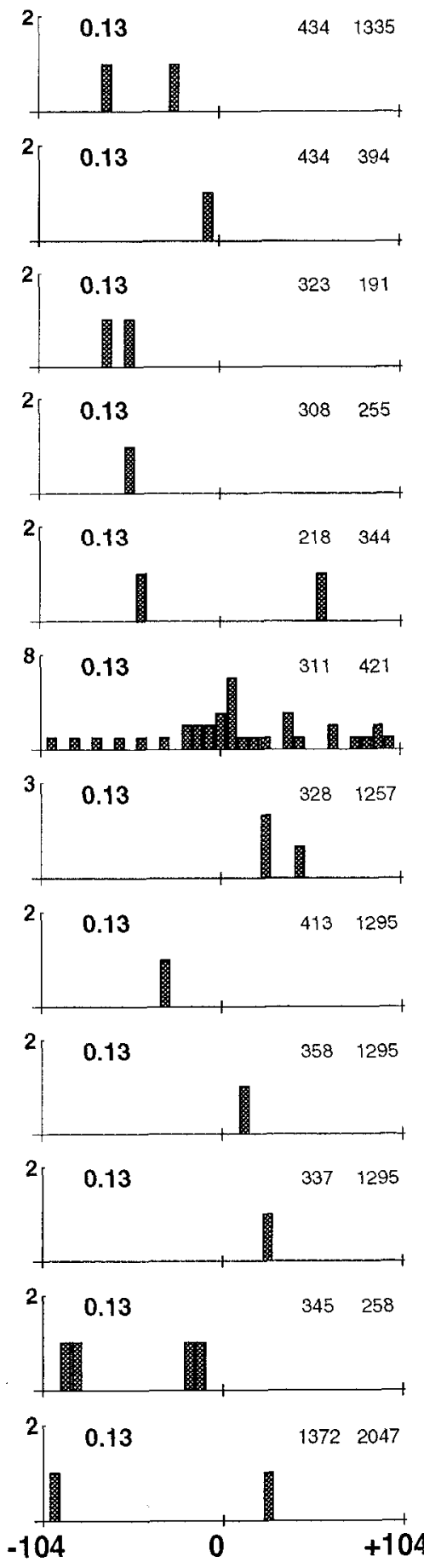

TIME (MSEC)
Figure 6. Twenty-four cross-correlograms from experiment $A 206$. In the left panel, the correlograms for the 12 neuron pairs with the largest correlation coefficients are presented. In the right panel, 12 pairs with correlation scores between 101st and 112th largest are shown. Each bar represents a time interval of $6.5 \mathrm{msec}$. The correlation coefficient is indicated in the upper left of each correlogram. The ID numbers of the neuron pairs are given in the upper right of each panel. The bin width used for the calculation of the correlation coefficient was 12 data points (three bins). The raster diagrams of some of the neurons illustrated in the correlograms can be located in Figure 2 of Wu et al. (1994); because neurons that made less than one spike per trial were omitted from the Figure 2, not all the neurons in the correlograms can be found in Figure 2.
To compare the correlation coefficients from the experimental and random data sets we have plotted the cumulative number of pairs with coefficients larger than given values versus the correlation coefficients. The top panel of Figure 8 is a plot of this data from an experimental data set (A207) and from the 10 randomized data sets. The diamonds are the results from the experimental data set, the triangles are the largest cumulative number from any of the 10 randomized sets, and the circles are the smallest number from any of the 10 . For all three curves the number of pairs increases rapidly with smaller correlation scores. The vast majority of both the experimental and randomized pairs have correlation coefficients of 0.1 or less. This is the expected result if most of the correlations were due to chance. However, even though the three curves are similar, the curve for the experimental data set (diamonds) is slightly higher than both curves (triangles and open circles) from the random- 
1-12; RANDOM

corr. coeff.
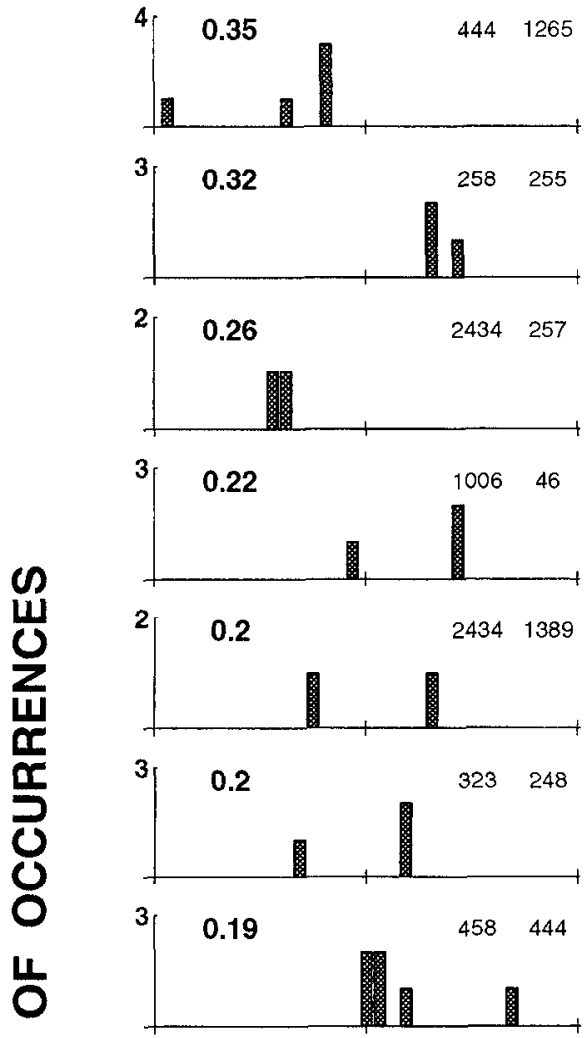

$\frac{1}{2}$
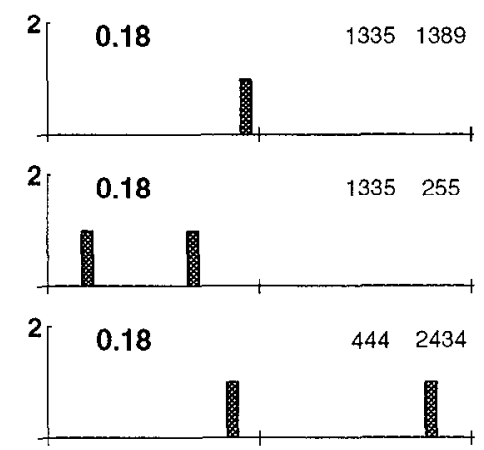

Figure 7. Twenty-four cross-correlograms from a randomized data set created from the data of cxpcriment A206. The cross-correlograms from those pairs generally had smaller scores and smaller number of occurrences in comparison with the corresponding cross-correlograms from the experimental data (Fig. 6). For Figure 7 we selected the randomized data set with the largest correlation coefficients. The organization of the figure is the same as Figure 6.

\section{1-112; RANDOM}
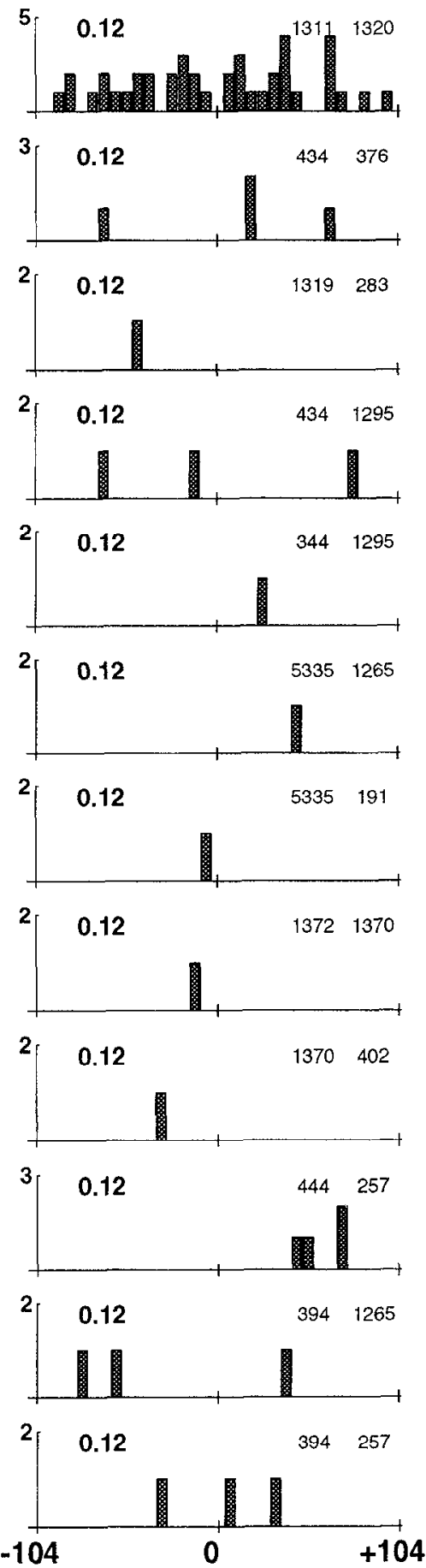

TIME (MSEC) ized data sets. This is shown in the middle panel of Figure 8 , where the same data is replotted beginning with a correlation coefficient of 0.2 and using an expanded y-axis. It is clear that the curve from the experimental data set is higher than the largest of the 10 randomized data sets. Thus, in this experiment there is a significant excess of large correlation coefficients in the experimental data.
For the bottom panel of Figure 8 the mean of the number of pairs from the random data sets was subtracted from both the largest of the random numbers and from the experimental numbers. As expected from the middle panel, there are more pairs in the experimental data set than in the largest of the random data sets over the range displayed. Figure 9 is identical to the bottom panel of Figure 8 but shows the means and SEs from 

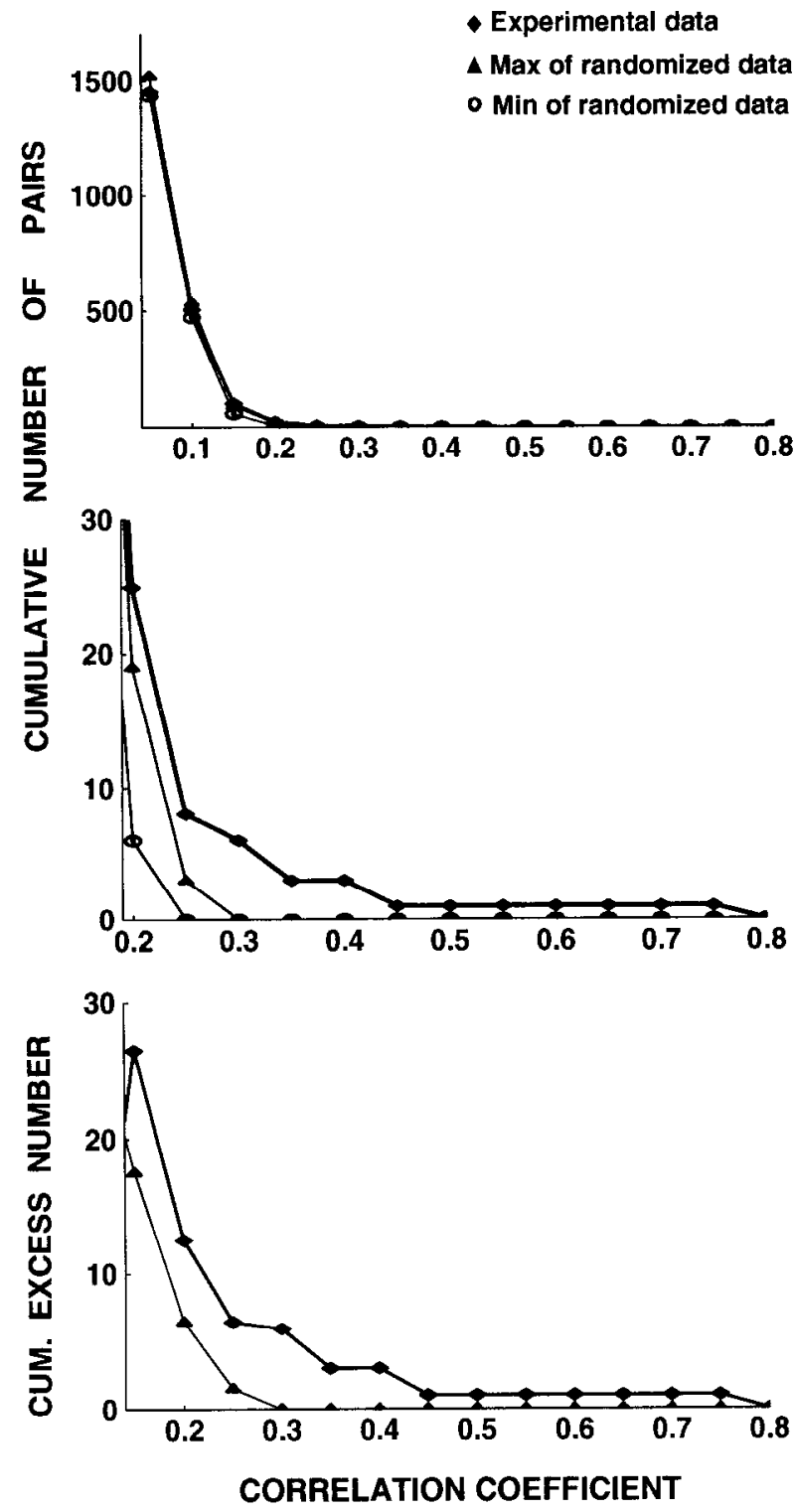

Figure 8. Plots of the cumulative number of pairs with correlation coefficients larger than the values on the abscissa. Numbers of pairs from three data sets are shown: the diamonds indicate values from the experimental data, the triangles are largest values from the 10 random data sets, and the circles are the smallest values from the random data sets. Top, There is a steep and monotonic decline in number of pairs with increasing correlation coefficient for all three curves, suggesting that a large majority of the correlations are due to chance occurrences. Middle, The same data with expanded $x$ - and $y$-axes. The number of pairs from the experimental data is larger than the largest numbers from the random data sets at all correlation coefficients shown. There is an excess of pairs in the experimental data set. Bottom, The number of excess pairs in the experimental data set and in the randomized data set with the largest scores. The mean number of pairs in the 10 randomized data sets was subtracted from the from the top two curves in the middle panel to show the excess of the number of pairs. Data from experiment A207 (Fig. 4).

four data sets. There is a significant excess of pairs with large correlation coefficients in the experimental data sets. At a correlation coefficient of 0.3 the number of excess pairs is about 5 . This number increases by an additional 25 pairs as the scores decrease to 0.15 . At correlation coefficients of 0.05 or 0.10 , the number of pairs in the experimental data was smaller than the

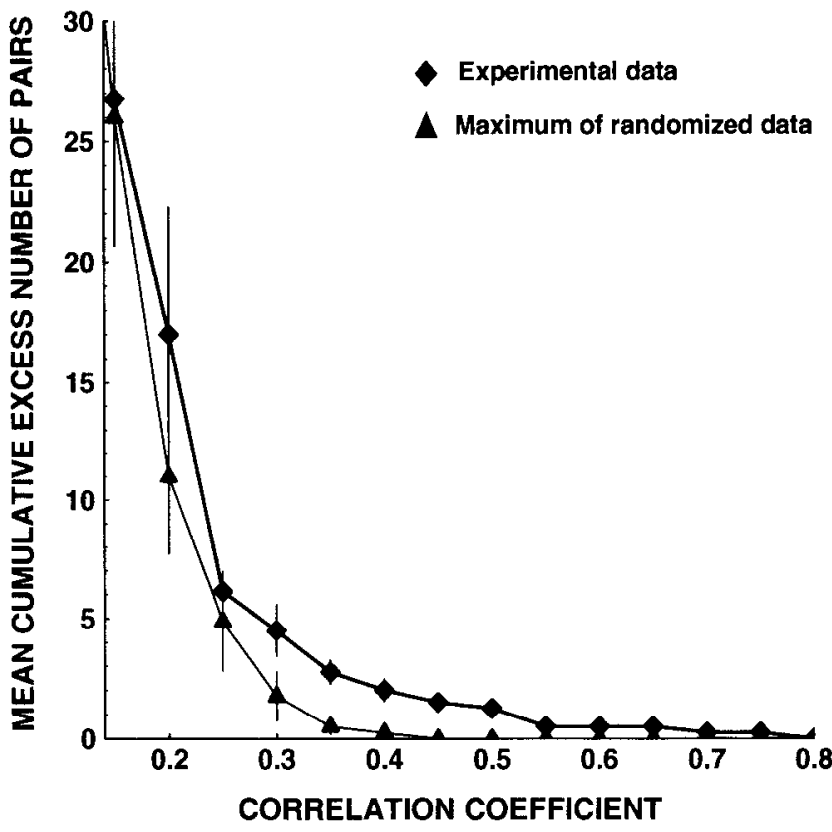

Figure 9. The mean values of the excess number of pairs in experimental data sets and random data sets with the largest number of pairs from the first four experiments of Table 2 (A188, A191, A206, and A207). There is a mean excess of about five pairs in the experimental data set at a correlation coefficient of 0.3 ; about 20 additional pairs are added between 0.25 and 0.15 . The correlation coefficients for Figures 8 and 9 were calculated using the maximum number of occurrences in any 12 data point window in the interval surrounding each spike. A similar number of excess pairs was also obtained when we used the maximum number in 4 or 20 data point windows. In addition, the result was substantially similar using total intervals surrounding each spike of $\pm 16, \pm 32, \pm 64$, and \pm 128 data points. For Figures 8 and 9 we did not include neurons with a mean of less than 0.5 spikes per trial. A similar number of excess pairs was obtained when all neurons were included or when we excluded neurons with a mean of less than 1.0 spikes per trial.

maximum from the randomized data sets (data not shown). Thus, we did not detect an excess of these small correlations in the experimental data. In an examination of correlations scores among approximately 10,000 pairs of neurons, we found that there were an excess of about 5 pairs $(0.05 \%)$ with correlation coefficients of greater than 0.3 and about 25 pairs $(0.2 \%)$ with scores between 0.15 and 0.25 .

We also constructed three-dimensional cross-correlograms to search for instances where the spikes in one neuron were correlated with simultaneous activity in two presynaptic neurons. No significant correlations of this kind were detected.

\section{Slow correlations}

Inspection of Figures 4 and 5 makes it clear that, over time intervals of seconds, the spike activities of a large fraction of the neurons are highly correlated. In both experiments there is a group of neurons that are active together just following the stimulus and a group that is active about $5 \mathrm{sec}$ later. The top section of Figure 10 illustrates 15 correlograms with a longer time base $( \pm 3.3 \mathrm{sec})$ made from neurons illustrated in Figure 5. These cross-correlograms were made using all 15 possible pairs among six neurons with a large number of spikes in the last $6.5 \mathrm{sec}$ of the recordings. With this time scale the crosscorrelograms of all possible cell pairs have many correlated spikes. In the experiment of Figure 5 there were about 140 pairs 


\section{Cross-Correlograms On A Long Time Scale}
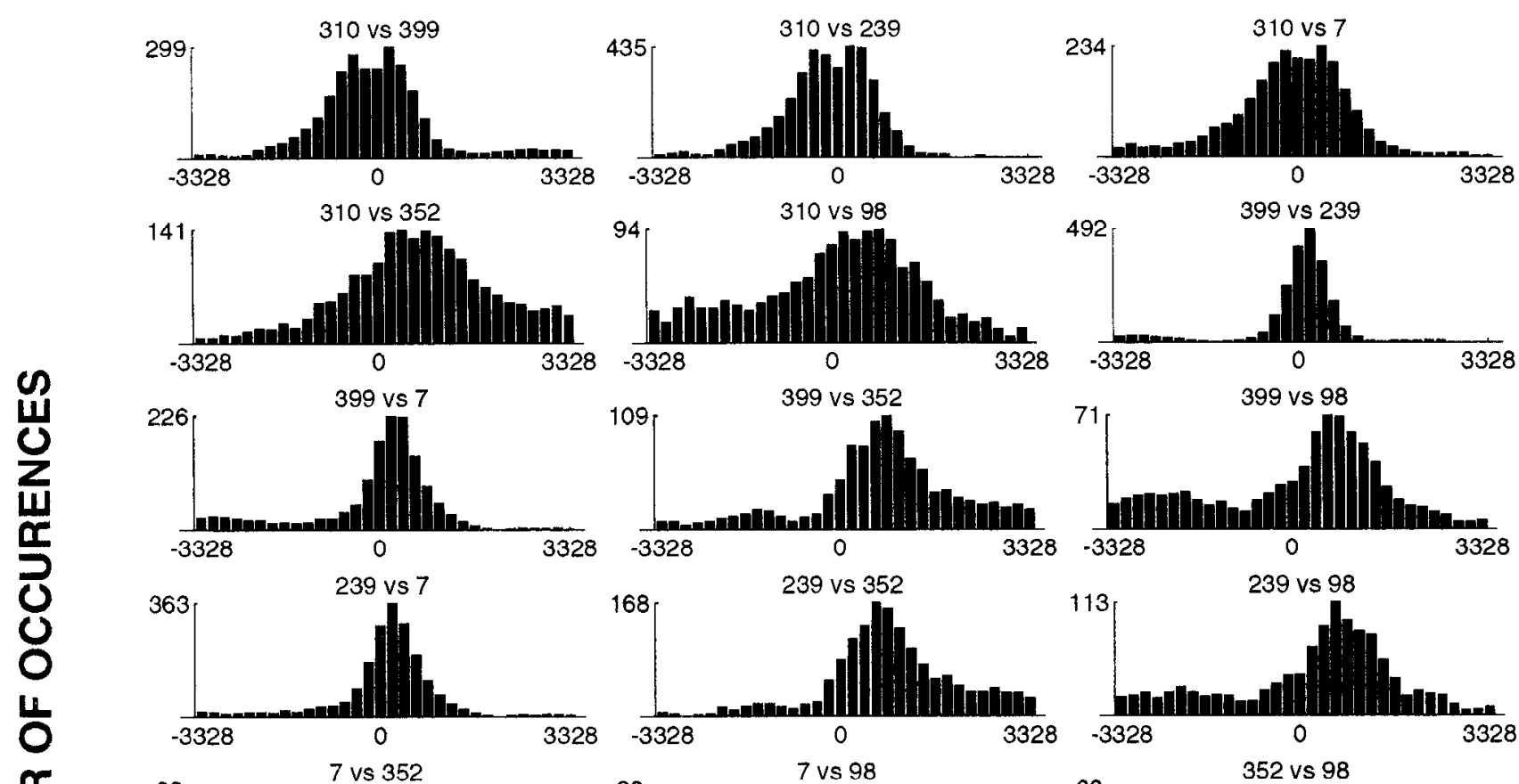

䍃
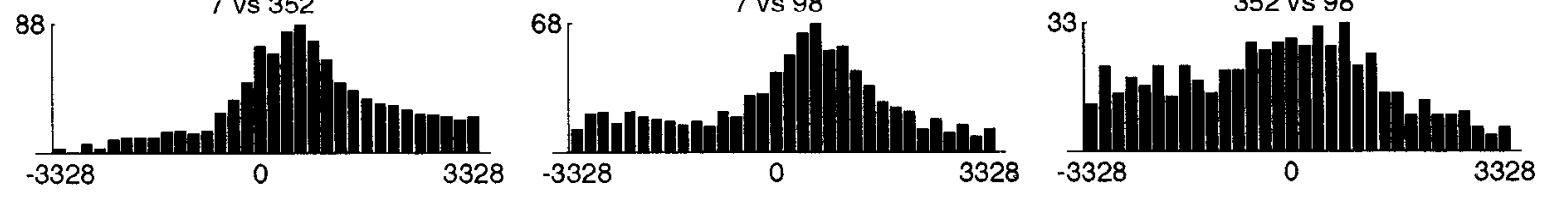

\section{Shifted Data}
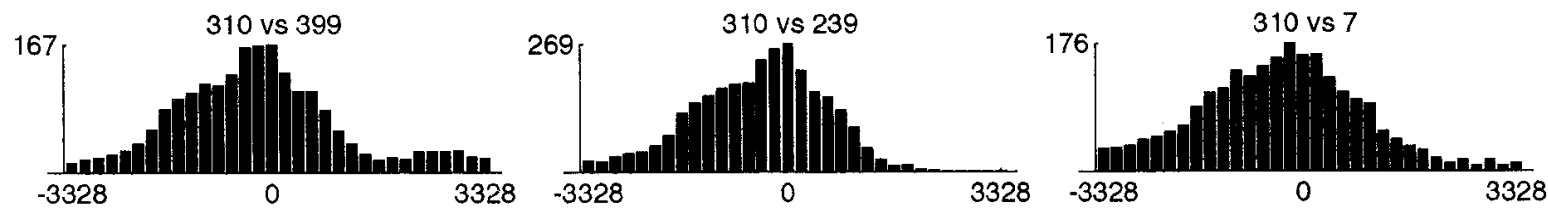

TIME DELAY (MSEC)

Figure 10. Top, Fifteen cross-correlograms on a slow time scale from the data illustrated in Figure 5 (experiment A217). All of the pairs have significant correlations using the slow time scale. The figure shows all possible pairs of neurons from six neurons with a large number of spikes in the last $6.5 \mathrm{sec}$ of the recording (late response). Bottom, The correlograms from the top three pairs of neurons were recalculated after shifting the spike times of one of the pair of neurons by one trial. The shifted correlograms are similar although slightly broader.

with a similar large number of correlated spikes in their slow cross-correlograms; about 1000 pairs of neurons from the experiment illustrated in Figure 4 had slow correlations.

Because the activity of most neurons is similarly correlated with the stimulus in each trial, we expected that cross-correlograms constructed from shifted data would also have a large number of correlated spikes. For the top three pairs from Figure 10 we computed the cross-correlograms after shifting the data from one of the two neurons by one trial. The results are shown at the bottom of Figure 10. As anticipated, the shifted correlograms have a large number of correlated spikes even though these correlograms are slightly broadened as a result of trial-totrial variability in the responses.
Possible connections between the neurons illustrated in Figures 5 and 10 are discussed below.

\section{Discussion}

The spread of sensory information in the Aplysia CNS

A large number of neurons in the abdominal, pedal, and pleural ganglia respond to the very mild and localized siphon stimulus (Fig. 3). No responses to siphon touch were detected in the cerebral ganglion. The third column of numbers in Table 1 gives the estimated normalized proportion of neurons in these ganglia that are affected by the stimulus. To estimate the actual numbers of neurons affected by the siphon stimulus we need to make two adjustments. The first is to correct for the incompleteness 


\section{DEDICATED CIRCUIT}

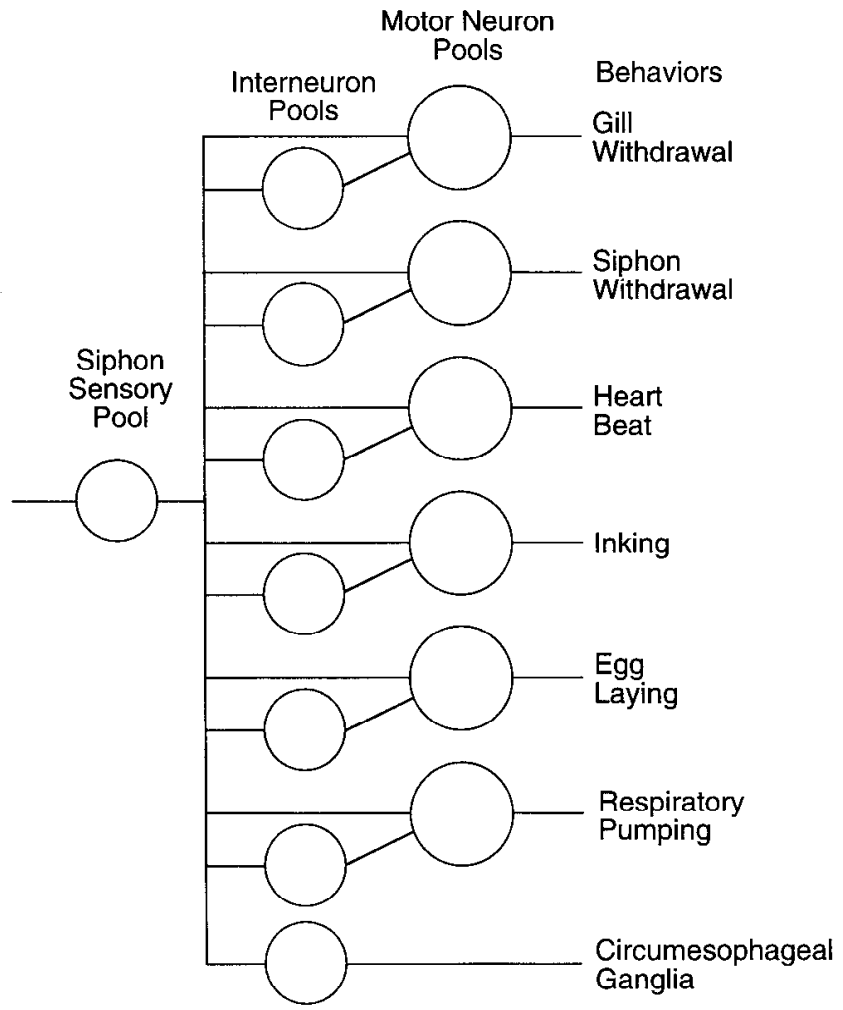

\section{DISTRIBUTED CIRCUIT}

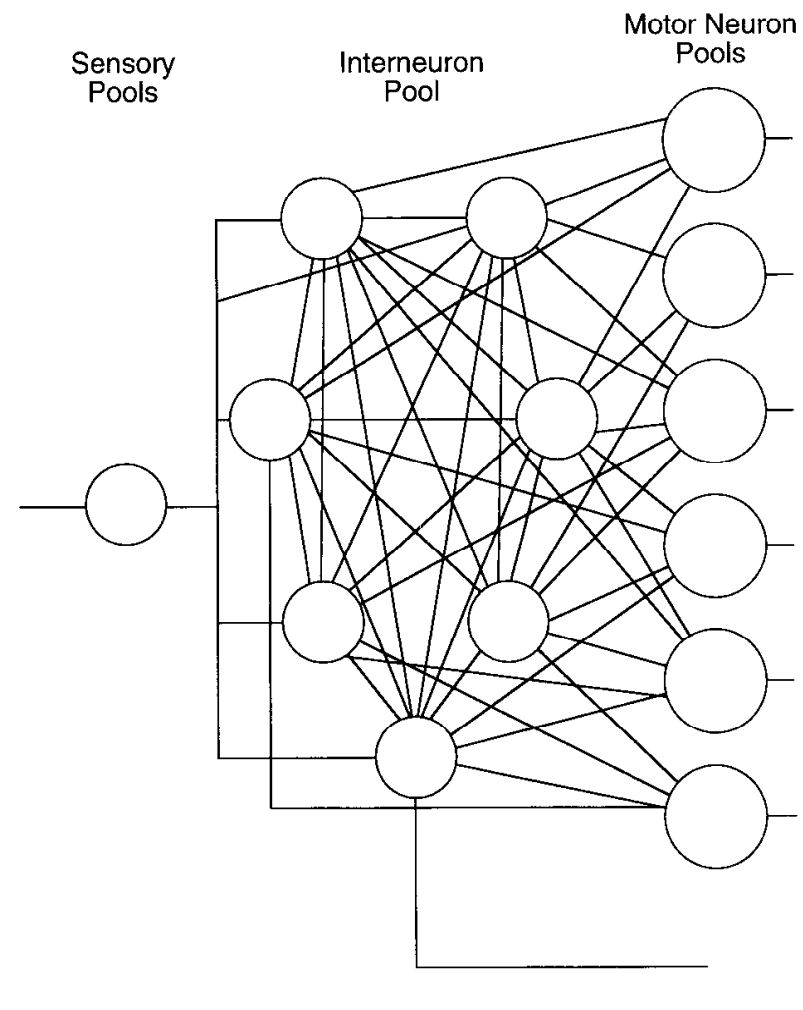

Figure 11. Schematic comparison of dedicated and distributed circuit organizations for the Aplysia abdominal ganglion. In both circuits a large number of neurons could be activated by a sensory stimulus but the convergence of information onto motor neurons will be larger in the distributed circuit.

of the voltage-sensitive dye recordings. Wu et al. (1994) estimated that the most complete recordings detected the activity of about $50 \%$ of the neurons in the abdominal ganglion. In the absence of information about the completeness of the recordings in the other central ganglia, we assume that they are also $50 \%$ complete. Second, we think that the experiment illustrated in Figure 3 was not optimal. In other voltage-sensitive dye recordings from abdominal ganglia, the number of activated neurons has ranged from a low of near zero to a high of 135 . Because there are many factors that could cause the number of detected neurons to be artifactually reduced (incomplete staining, excess optical noise, preparation in poor condition), our prejudice is that the larger numbers are more representative of normal neuron activity. If we take 110 neurons as the mean number that would be detected in "optimal" measurements, correct for completeness, and use the proportions in the third column of Table 1 , then the actual number of abdominal neurons that were activated was 220 , the number of pedal neurons was 650 , and the number of pleural neurons was 110 . The total number of affected neurons is thus approximately 1000 . The last column of Table 1 indicates the percentages of the neurons in the three ganglia that responded to the touch. These percentages were calculated using the number of neurons in these ganglia determined by Coggeshall (1967) and Cash and Carew (1989). An unknown number of additional neurons are likely to receive subthreshold excitatory or inhibitory inputs.

The estimate of 1000 affected neurons relies on several assumptions and estimations. The most important assumption is that there was symmetrical activation of neurons in the paired pedal and pleural ganglia. We needed to make an assumption since we measured the activity in only one of each of the pairs. Our best estimate is that about $25 \%$ of the 900 neurons in the abdominal ganglion and $15 \%$ of all 8000 central neurons (Cash and Carew, 1989) respond to this modest stimulus. Information about light touch is very widely distributed within the Aplysia CNS. Although our estimate of the number of neurons has a number of uncertainties, it may be good in comparison to estimates made with other methods. Optical recordings have the advantage over 2-deoxyglucose estimates (e.g., John et al., 1986) in that the timing of the neuron activity relative to the behavioral output is precisely determined.

In the isolated siphon preparation with no circumesophageal ganglia, the only movement that occurs reliably in response to a light siphon touch is gill withdrawal (H.-P. Hopp, unpublished observations). However, in a more intact preparation that includes the circumesophageal ganglia, there are additional parapodial and mantle shelf movements (Kandel, 1979; Hopp, unpublished observations). Some of the estimated 750 neurons in the pedal and pleural ganglia that are activated by the touch are likely to be responsible for generating and coordinating these additional components of the response.

\section{Cross-correlations as an indicator of neuron interactions}

Figure 11 illustrates the distinction between a dedicated circuit and a distributed organization for the Aplysia abdominal ganglion. Even though the number of "cells" is the same in both models, the number of neuron interactions is larger in the distributed model. Thus, one might expect that the synaptic strengths 


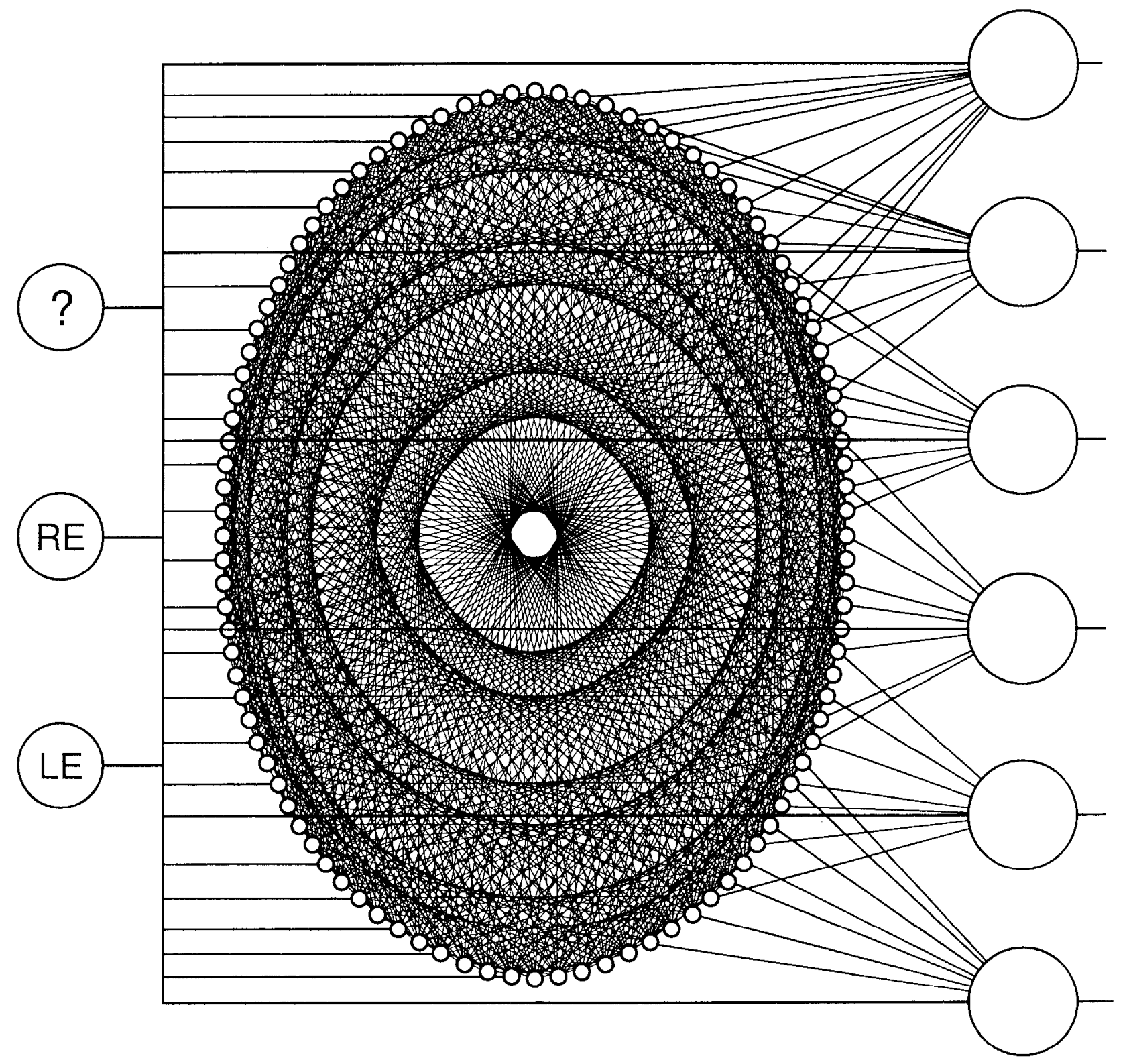

Figure 12. Schematic diagram illustrating the number of neurons and synaptic interactions involved in the gill withdrawal reflex based on results from Aplysia and C. elegans. One hundred interneurons are illustrated and each interneuron makes synaptic contact with $8 \%$ of all the other interneurons. Twice this number of lines emanate from each interneuron, eight for the synapses made by each interneuron and eight for the synapses made on to the neuron. The complexity of the interactions is evident. In a distributed system these interneurons would also be involved in generating other behaviors.

in a distributed system would be smaller than those in a dedicated system. One indirect measure of synaptic strength is the amount of correlation in the timing of action potentials of pairs of neurons.

Fast correlations. Carrying out the cross-correlation analysis during the response to a sensory stimulus has two consequences. First, the correlation strengths were measured while the gillwithdrawal reflex was generated so that the correlation coefficients reflect the situation in the nervous system during the response rather than during a quiescent period where the physiological characteristics of the neurons (e.g., response to a unit synaptic input) might be quite different. Second, it is clear from the large amount of spike activity generated by the stimulus that the membrane potentials of many neurons must have been near threshold for some fraction of the poststimulus interval. This would increase the likelihood that relatively small synaptic potentials could affect the firing of the postsynaptic neuron, thereby increasing the likelihood of finding large correlation coefficients.

However, the cross-correlation analysis indicated that there was only a small number of excess pairs with large correlation coefficients in the experimental data sets. The number was a 
tiny fraction of the total number of pairs examined: $0.2 \%$ at a score of $0.15-0.25$, and $0.05 \%$ at a score of $>0.3$. Thus, interactions that are excitatory, fast, and large enough to generate a correlation coefficient of 0.15 or greater are very rare among the neurons that generate the response to siphon touch. On the other hand, very mild sensory stimulation activates approximately 1000 neurons; presumably this occurs via excitatory interactions that are small and/or slow. From the data in Table 2 and Гigure 9 we estimate that less than $3 \%$ of the action potentials in the response result from the neuron pairs with correlation coefficients of 0.15 or greater.

Our finding that most correlation strengths are small can be compared with measured synaptic strengths in Aplysia. The size of the EPSP made by LE sensory neurons onto motor neurons ranges from less than $0.1 \mathrm{mlV}$ to more than $6 \mathrm{mV}$ (Byrne et al., 1978; Frost et al., 1985; C. Hickie and T. Walters, personal communication). Hickie and Walters obtained a mean size of $2.5 \mathrm{mV}$ and a mean width at half-height of $70 \mathrm{msec}$ in measurements from 54 different pairs of LE sensory neurons and siphon motor neurons. Thus, the mean area under the synaptic potential from these individual presynaptic neurons was 175 $m V * m s e c$ (range $<5-500$ ). This can be compared with an estimate of the area under the motor neuron EPSP in response to a light touch, which averaged $35,000 \mathrm{mV} * \mathrm{msec}$ (rangc, 20,000$80,000)$ in measurements from Figures 3-5 and 12 of Byrne et al. (1978). Thus, each action potential in an individual LE neuron would contribute approximately $0.5 \%$ of the total postsynaptic effect. The very small contribution of an individual LE neuron is consistent with our finding of very few large correlation coefficients. The finding that large fast synaptic connections seem to be relatively unimportant is consistent with the expectation that there would be a preponderance of small synaptic effects in a distributed system. If the synaptic effects of individual neurons are small, then the presynaptic activity of many neurons are required to activate a postsynaptic neuron.

No fast inhibitory interactions were detected in the crosscorrelograms. This may not be surprising since the detection of inhibitory interactions using cross-correlation analysis is strongly dependent on spike frequency (Aersten and Gerstein, 1985). The average mean frequency (Table 2 ) was only $2 / \mathrm{sec}$, and thus inhibitory interactions would be difficult to detect.

Differentiating those pairs that are the real excess from those that occurred by chance may not be possible. There was a mean excess of about five pairs with scores of greater than 0.3 and an additional mean excess of 25 pairs with scores that were between 0.15 and 0.25 . These are excesses over the mean number of pairs found in the random data sets. In the random data there was a mean of 180 pairs at a score of 0.15 and a mean of 2 pairs at 0.3 . Thus, only a sixth of the pairs in the experimental data set with scores of greater than 0.15 are in excess. About two-thirds of the pairs with scores greater than 0.3 are in excess. Unfortunately, we do not know how to determine which of the individual pairs belong to the group that is in excess and which belong to the group that occurred by chance.

Both the small number of pairs with detectable correlations and the difficulty in identifying which of those pairs were real and which were due to chance means that the cross-correlation methods we have used cannot provide information about the large number of synaptic interactions (see below) that must underlie the generation of behaviors in Aplysia. There are, however, two caveats. First, it is possible that more complex interactions might be detected by further analyses. Second, this con- clusion may be specific to data sets that, like the optical recordings, have a limited duration $(\sim 100 \mathrm{sec})$ and therefore a relatively small number of spikes.

Slow correlations. As anticipated from the fact that most spike activity is stimulus locked, a large number of pairs of neurons have correlated activity measured on a slow time scale. The right panels in Figure 5 show the locations on the photodiode array for two groups of neurons from the experiment illustrated in the left panels. The top right panel shows the locations for the neurons that were active during the time when the stimulated neuron (\#283) was driven to make its train of 19 spikes (early response). The bottom panel shows the locations of the neurons that were active during the second burst about $6 \mathrm{sec}$ after the stimulation (late response).

In a situation where many pairs of neurons have slow correlations in their activity, it is not possible to determine which pairs of neurons have synaptic connections and which pairs have correlated activity that arises from indirect, polysynaptic connections. We have arbitrarily selected two plausible synaptic interactions and used them the right panels of Figure 5. One simple possibility for the synaptic organization of the early response group is that there is one driver neuron and all other neurons in the group are followers. The drawing on the top right of Figurc 5 illustrates this possibility. More complex interactions are shown in the bottom right panel of Figure 5. In Figure 10 some cross-correlograms are symmetrical about time 0 , implying that the time course of the burst of activity in the pair was identical. However, many of the correlograms are asymmetric, which results from the fact that the burst of spikes in these pairs were not temporally coincident. From each cross-correlogram a relative temporal order can be determined for the two cells. From all of these comparisons the cells can be ordered according to their relative firing times. One simple possibility for the synaptic organization of the late response group of neurons is that it follows this temporal ordering. The synaptic connections based on this ordering are shown in the bottom right panel of Figure 5 . Unfortunately, these are only plausible synaptic interactions; the cross-correlation results do not allow us to distinguish these two schemes from a large number of plausible schemes.

The finding that there are large number of slow correlations is consistent with the suggestion that slow synaptic interactions may be important in generating the Aplysia gill-withdrawal reflex. However, slow correlations could also be generated by the sum of many small, relatively brief synaptic interactions.

\section{The Aplysia CNS as a distributed system}

The results in Figure 3 and Table 1 show that information about touch is very widely distributed in the Aplysia CNS. Approximately 1000 neurons in the abdominal, pleural, and pedal ganglia are activated by the siphon stimulus. This defines the number of neurons active during the defensive withdrawal in the reduced preparation we used. However, the observation that a neuron is active does not demonstrate that it actually participates in generating a particular behavior; some of the neurons may be part of circuits for other behaviors that are initiated and/or modulated by the sensory stimulus. For cxamplc, the finding that there are a large number of activated neurons in the abdominal ganglion will not distinguish the dedicated (on the left) and distributed (on the right) circuit architectures illustrated in Figure 11. In both schemes a large number of neurons are activated by the sensory input but in the dedicated circuit only a portion of the activated neurons contribute to gill 


\begin{tabular}{|c|c|c|c|c|}
\hline $\begin{array}{l}\text { Sensory } \\
\text { neurons }\end{array}$ & $\begin{array}{l}\% \text { Mono- } \\
\text { synaptic }\end{array}$ & $\begin{array}{l}\% \text { Di- } \\
\text { synaptic }\end{array}$ & $\begin{array}{l}\% \text { Mono- } \\
\text { synaptic }\end{array}$ & $\begin{array}{l}\text { Motor } \\
\text { neurons }\end{array}$ \\
\hline ADE-L & 9 & 61 & & \\
\hline OLL-L & 5 & 36 & & \\
\hline PHC-L & 2 & 31 & & \\
\hline \multirow[t]{4}{*}{ ADL-L } & 7 & 54 & & \\
\hline & & 37 & 8 & RMD-DL \\
\hline & & 30 & 2 & RID \\
\hline & & 59 & 7 & RIM-L \\
\hline
\end{tabular}

withdrawal. One difference between the two schemes is that the dedicated circuit has a large divergence from the sensory neurons but a relatively restricted convergence onto the motor neurons. On the other hand, the distributed circuit has both a large divergence from sensory neurons and a large convergence onto motor neurons. Our data only provide information about the divergence from the sensory inputs; they do not provide information about convergence onto motor neurons.

However, information about the relative extents of divergence and convergence can be obtained from another preparation. The serial section electron microscopy data of White et al. (1986) has provided a complete anatomical map of the synaptic connections in the Caenorhabditis elegans nervous system. The divergence from sensory neurons and convergence to motor neurons can be directly compared using this data. Table 3 shows the percentage of the neurons in the animal that are postsynaptic to four sensory neurons and the percentage that are presynaptic to three motor neurons. The four sensory neurons make monosynaptic connections to about $6 \%$ of all the animal's neurons. These neurons in turn make synaptic connections with about $50 \%$ of all the neurons. Thus, after two synapses the sensory information will have reached about $50 \%$ of all the neurons in C. elegans. The three motor neurons receive synaptic input from about $6 \%$ of all the animal's neurons; these cells in turn receive input from about $50 \%$ of the neurons. In $C$. elegans the divergence and convergence are very symmetrical. This symmetry may also hold in Aplysia although there is at present no direct evidence on this point. If Aplysia is also symmetrical, then a very large number of neurons would affect the spike decisions of each motor neuron, a result that is consistent with the distributed circuit illustrated in the right panel of Figure 11.

Byrne et al. (1978) reported that the monosynaptic connection made by LE scnsory ncurons accounted for $58 \%$ of the excitatory input to the gill motor neurons. If true, this result would argue strongly for an important dedicated component and a simple architecture. However, results available at that time, as well as more recent findings, suggest that $58 \%$ greatly overestimates the LE contribution. First, each LE sensory neuron makes only one or two action potentials in response to a light touch (Byrne et al., 1978). This is in contrast to touch neurons in leech segmental ganglia, which fire a brisk train of spikes in response to the same stimulus (Nicholls and Baylor, 1968). The modest response to touch of the LE neurons suggested that other neurons might be important in transducing information about light touch. Second, the total LE contribution to the motor neuron PSP can be estimated. Each touch was said to activate eight LE sensory neurons (Byrne et al., 1978). From the estimate (see above) that each LE action po- tential accounts for about $0.5 \%$ of the motor neuron response, the total LE sensory neuron contribution to the motor neurons EPSP is about $5 \%$, an order of magnitude smaller than the estimate of Byrne et al. (1978).

More recently, Trudeau and Castellucci (1992a) reexamined the effects of the divalent cation solution that had been used (Byrne et al., 1978) to block interneuron activity. Trudeau and Castcllucci (1992a) found that this solution increased the size of a monosynaptic EPSP. With a divalent cation solution that did not have this effect, the total monosynaptic contribution was only $23 \%$. Even this smaller value is likely to be an upper limit for the actual monosynaptic contribution. Several of the interneurons (L16 and L30) whose activity is blocked by the high-divalent solution are inhibitory (Frost, 1987). Thus, the monosynaptic EPSP remaining in high-divalent seawater may be exaggerated because of the loss of this inhibition. In addition, the polysynaptic EPSP has a peak membrane potential closer to the equilibrium potential and thus potential measurements will tend to underestimate the polysynaptic conductance change (Byrne et al., 1978). Furthermore, the LE sensory neuron contribution to this monosynaptic component is not certain. Cohen et al. (1991) found that LE neurons always fired after the motor neurons in simultaneously recorded pairs of LE sensory neurons and motor neurons. Finally, the LE ncurons are not activated by light touches that do activate the gill-withdrawal reflex (Fischer and Carew, 1993; Kaplan et al., 1993). Thus, the LE neurons provide only a small fraction (0-10\%) of the excitatory drive for the gill motor neurons in the response to a light touch. Simple, monosynaptic connections account for only a small fraction of the behavior.

Weaknesses in the argurnents for a distributed system. We have presented arguments from three kinds of evidence to support the notion that the abdominal ganglion functions in a distributed manner. Each argument has weaknesses. The first argument came from the combined results that the divergence of sensory information in Aplysia was dramatic and that divergence and convergence in $C$. elegans were symmetrical. Assuming that the symmetry seen in $C$. elegans would also be found in Aplysia, one can conclude that a large number of neurons would also participate in determining the activity of Aplysia motor neurons. However, the validity of this assumption is unknown; the nervous systems of Aplysia and C. elegans may be organized differently. The second argument came from the finding that only a small fraction of the spikes that result from siphon touch are generated by cell pairs with large correlation coefficients, suggesting that the synaptic strengths of most neuron interactions are weak. This argument is not strong because we do not know the relationship between correlation coefficient and synaptic strength. Furthermore, we cannot make a quantitative prediction of the expected correlation coefficients from either the dedicated or the distributed model and thus only qualitative conclusions can be drawn. The third argument came from the extensive overlap of the neurons used during two different behaviors generated by the abdominal ganglion ( $\mathrm{Wu}$ et al., 1994). This argument is weak because the two behaviors are not very different and we do not know the proportion of motor neurons and interneurons in the optical recordings. There is the possibility, albeit unlikely, that the majority of neurons that we record are motor neurons. Thus, our arguments in favor of a distributed system for generating the Aplysia gill-withdrawal reflex are far from compelling. Nonetheless, taken together, they suggest that the notion of a distributed organization may be 
important in characterizing the neuronal substrate of the gillwithdrawal reflex.

Arguments against a distributed system. One kind of observation that argues against a completely distributed organization is the existence of interneurons with specific function. Electrophysiological measurements have suggested the existence of 22 interneuron types (composed of a total of 65 neurons) that are involved in withdrawal reflexes (Koester and Kandel, 1977; Byrne and Koester, 1978; Byrne, 1980, 1983; Hawkins et al., 1981; Frost, 1987; Alevizos et al., 1989a,b; Koester, 1989; Trudeau and Castellucci, 1992b; Koester et al., 1993; J. Koester, unpublished observations). If each of these interneuron groups has distinct follower neurons and distinct functions in the generation of the gill withdrawal, then one could conclude that the gill-withdrawal reflex is generated by a relatively dedicated organization of neurons. On the other hand, if these interneuron groups have overlapping followers and overlapping functions, then a distributed organization would be favored. Additional experiments will be required to determine the extent to which a distributed organization can account for the generation of the Aplysia gill withdrawal.

\section{A schematic diagram}

In Figure 12 we present a schematic drawing of a circuit for the gill-withdrawal reflex. Four aspects of the figure are based on information from the Aplysia and $C$. elegans nervous systems.

(1) One hundred interneurons are illustrated. Microelectrode recordings have already demonstrated the existence of more than 60 interneurons involved in defensive withdrawals. However, only 230 neurons in the ganglion have been thus far idenlified (J. Koester, personal communication) out of a total of about 900 neurons. If interneurons make up the same proportion of the remaining unidentified neurons, the total number of interneurons will exceed 200 . Thus, the number of interneurons illustrated in Figure 12 is reasonable.

(2) Each interneuron makes a synaptic contact with $8 \%$ of all other interneurons. This percentage interaction was obtained from a compilation from White et al. (1986), which showed that five $C$. elegans interneurons contacted a mean of $8 \%$ of all neurons in the animal. Individual Aplysia neurons make more than 1000 anatomical synapses (Bailey and Chen, 1988); thus, $8 \%$ is not unreasonable for Aplysia. If the interaction percentage in Aplysia were $8 \%$, then the number of synaptic interactions in the abdominal ganglion would be more than 50,000. The number shown in Figure 12 is less than 1000.

(3) The fraction of interneurons synaptically activated by sensory neurons in Figure 12 was taken from the C. elegans results. The LE sensory neurons in Aplysia actually activate a larger fraction of the known Aplysia interneurons.

(4) Finally, the percentage of the motor neuron input that arises from monosynaptic connections from sensory neurons is not far from the estimate from Aplysia.

Thus, we have attempted to be numerically realistic in making the schematic drawing in Figure 12. However, the schematic drawing is seriously, and misleadingly, simplified in implying that all of the interneurons are identical. Both microelectrode and optical recordings suggest that the neuronal substrate of the Aplysia gill-withdrawal reflex is neither simple nor well understood.

Because the activity of a large fraction of the neurons can be monitored with voltage-sensitive dyes, it seems possible that the Aplysia preparation will be unusually favorable for deter- mining where its organization lies in the spectrum between fully dedicated and fully distributed.

\section{References}

Aertsen AMHJ, Gerstein GL (1985) Evaluation of neuronal connectivity: sensitivity of cross correlation. Brain Res 340:341-354.

Alevizos A, Weiss KR, Koester J (1989a) SCP-containing R20 neurons modulate respiratory pumping in Aplysia.. J Neurosci 9:3058-3071.

Alevizos A, Weiss KR, Koester J (1989b) Neurosecretory cell R 15 activates respiratory pumping, motor neuron $\mathrm{L} 7$, and the hermaphroditic duct in Aplysia. Soc Neurosci Abstr 15:1140.

Bailey CH, Chen M (1988) Long-term memory in Aplysia modulates the total number of varicosities of single identified sensory neurons. Proc Natl Acad Sci USA 85:2373-2377.

Byrne JH (1980) Neural circuit for inking behavior in Aplysia californica. J Neurophysiol 43:896-911.

Byrne JH (1983) Identification and initial characterization of a cluster of command and pattern-generating neurons underlying respiratory pumping in Aplysia californica. J Neurophysiol 49:491-508.

Byrne JH, Koester J (1978) Respiratory pumping: neuronal control of a centrally commanded behavior in Aplysia. Brain Res 143:87105.

Byrne JH, Castellucci VF, Kandel ER (1978) Contributions of individual mechanoreceptor sensory neurons to defensive gill-withdrawal reflex in Aplysia. J Neurophysiol 41:418-431.

Cash D, Carew TJ (1989) A quantitative analysis of the development of the central nervous system in juvenile Aplysia californica. J Neurobiol 20:25-47.

Christakos CN (1984) Note on the estimation of the correlation function of neural spike trains. Biol Cybern 50:115-117.

Coggeshall RE (1967) A light and electron microscope study of the abdominal ganglion of Aplysia californica. J Neurophysiol 30:12631287.

Cohen LB, Falk CX, Schiminovich D, Wu JY, Falk R (1990) Crosscorrelations in the spike activity of neurons in the Aplysia abdominal ganglion during the gill-withdrawal reflex. Biol Bull 179:229.

Cohen TE, Henzi V, Kandel ER, Hawkins RD (1991) Further behavioral and cellular studies of dishabituation and sensitization in Aplysia. Soc Neurosci Abstr 17:1302.

Eggermont JJ (1990) The correlative brain. Berlin: Springer.

Falk CX, Wu Jy, Cohen LB, Tang C (1993) Non-uniform expression of habituation in the activity of distinct classes of neurons in the Aplysia abdominal ganglion. J Neurosci 13:4072-4081.

Fischer TM, Carew TJ (1993) Activity-dependent potentiation of recurrent inhibition: a mechanism for dynamic gain control in the siphon withdrawal reflex of Aplysia. J Neurosci 13:1302-1314.

Frost WN (1987) Mechanisms contributing to short- and long-term sensitization in Aplysia. PhD thesis, Columbia University.

Frost WN, Castellucci VF, Hawkins RD, Kandel ER (1985) Monosynaptic connections made by the sensory neurons of the gill- and siphon-withdrawal reflex in Aplysia participate in the storage of longterm memory for sensitization. Proc Natl Acad Sci USA 82:82668269.

Grinvald A, Cohen LB, Lesher S, Boyle MB (1981) Simultaneous optical monitoring of activity of many neurons in invertebrate ganglia using a 124-element photodiode array. J Neurophysiol 45:829-840.

Grinvald A, Manker A, Segal M (1982) Visualization of the spread of electrical activity in rat hippocampal slices by voltage-sensitive optical probes. J Physiol (Lond) 333:269-291.

Hawkins RD, Castellucci VF, Kandel ER (1981) Interneurons involved in mediation and modulation of the gill-withdrawal reflex in Aplysia. I. Identification and characterization. J Neurophysiol 45: 304-314.

John ER, Tang Y, Brill AB, Young R, Ono K (1986) Double-labeled metabolic maps of memory. Science 233:1167-1175.

Kandel ER (1979) Behavioral biology of Aplysia. San Francisco: Freeman.

Kaplan SW, Kandel ER, Hawkins RD (1993) Plasticity in the monosynaptic component of the Aplysia gill-withdrawal reflex during habituation, dishabituation and sensitization. Soc Neurosci Abstr 19: 16.

Kien J, Altman JS (1992) Decision-making in the insect nervous system: a model for selection and maintenance of motor programmes. In: Neurobiology of motor programme selection (Kien J, McCrohan CR, Winlow W, eds), pp 147-169. Oxford: Pergamon. 
Koester J (1989) Chemically and electrically coupled interneurons mediate respiratory pumping.in Aplysia. J Neurophysiol 62:11131126.

Koester J, Kandel ER (1977) Further identification of neurons in the abdominal ganglion of Aplysia using behavioral criteria. Brain Res 121:1-20.

Koester J, Alevizos A, Skelton M, Sullivan B (1993) Further examination of the functional role of neuron R15 in Aplysia. Soc Neurosci Abstr 19:18.

Kristan WB, Lockery SR, Wittenberg G, Brody D (1992) Making behavioral choices with interneurons in a distributed system. In: Neurobiology of motor programme sclection (Kien J, McCrohan CR, Winlow W, eds), pp 170-200. Oxford: Pergamon.

Kupfermann I, Pinsker H, Castellucci V, Kandel ER (1971) Central and peripheral control of gill movements in Aplysia. Science 174: 1252-1256.

Lindsey BG (1982) Measurement and dissociation of joint influence of action potentials in concurrently active parallel channels on motor neuron activity in crayfish. J Neurophysiol 47:1160-1173.

London JA, Zecevic D, Cohen LB (1987) Simultaneous optical recording of activity from many neurons during feeding in Navanax. $\mathbf{J}$ Neurosci 7:649-661.

Moore GP, Perkel DH, Segundo JP (1970) Statistical signs of synaptic interaction in neurons. Biophys J 10:876-900.

Nakashima M, Yamada S, Shiono S, Maeda M, Sato F (1992) 448Detcctor optical recording systcm: development and application to Aplysia gill-withdrawal reflex. IEEE Trans Biomed Eng 39:26-36.

Nicholls JG, Baylor DA (1968) Specific modalities and receptive fields of sensory neurons in the CNS of the leech. J Neurophysiol 31:740756.

Perkel DH, Gerstein GL, Moore GP (1967) Neuronal spike trains and stochastic point processes. I. Simultaneous spike trains. Biophys J 7:419-440.

Salzberg BM, Grinvald A, Cohen LB, Davila HV, Ross WN (1977) Optical recording of neuronal activity in an invertebrate central nervous system: simultaneous monitoring of several neurons. J Neurophysiol 40:1281-1291.

Schiminovich D, Cohen LB, Cohen AI, Hopp HP, Falk CX, Wu JY
(1989) A search for correlations in the spike activity of the Aplysia abdominal ganglion during the gill withdrawal reflex. Biol Bull 177: 325.

Tam DC, Ebner TJ, Knox CK (1988) Conditional cross-interval correlation analyses with applications to simultaneously recorded cerebellar Purkinje neurons. J Neurosci Methods 23:23-33.

Toyama K, Kimura M, Tanaka K (1981) Cross-correlational analysis of interneuronal connectivity in cat visual cortex. J Neurophysiol 46: 191-201.

Trudeau LE, Castellucci VF (1992a) Contribution of polysynaptic pathways in the mediation and plasticity of Aplysia gill and siphon withdrawal reflex: cvidence for differential modulation. J Ncurosci 12:3838-3848.

Trudeau LE, Castellucci VF (1992b) Functional uncoupling of cholinergic inhibitory neurons plays and important role in short-term sensitization of Aplysia gill and siphon withdrawal. Soc Neurosci Abstr 18:531.

White JG, Southgate E, Thomson JN, Brenner S (1986) The structure of the nervous system of the nematode Caenorhabditis elegans. Philos Trans R Soc Lond [Biol] 314:1-340.

Winlow W, Moroz LL, Syed NI (1992) Mechanisms of behavioral selection in Lymnaea stagnalis. In: Neurobiology of motor programme selection (Kien J, McCrohan CR, Winlow W, eds), pp 5272. Oxford: Pergamon

Wu JY, Cohen LB (1993) Fast multisite optical measurements of membranc potential. In: Fluorescent and luminesecnt probes for biological activity (Mason WT, ed), pp 389-404. London: Academic.

Wu JY, Cohen LB, Falk CX (1994) Neuronal activity during different behaviors in Aplysia: a distributed organization? Science 263:820823.

Wu JY, Tsau Y, Hopp HP, Cohen LB, Tang AC, Falk CX (1994) Consistency in nervous systems: Trial-to-trial and animal-to-animal variations in the response to repeated application of a sensory stimulus in Aplysia. J Neurosci 14:1366-1384.

Zecevic D, Wu JY, Cohen LB, London JA, Hopp HP, Falk CX (1989) Hundreds of neurons in the Aplysia abdominal ganglion are active during the gill-withdrawal reflex. J Neurosei 9:3681-3689. 